\title{
A yeast two-hybrid system for the screening and characterization of small-molecule inhibitors of protein-protein interactions identifies a novel putative Mdm2-binding site in p53
}

Jin Huei Wong ${ }^{1}$, Mohammad Alfatah', Mei Fang $\operatorname{Sin}^{2}{ }^{2}$, Hong May $\operatorname{Sim}^{2}$, Chandra S. Verma ${ }^{1,3,4}$, David P. Lane ${ }^{5}$ and Prakash Arumugam ${ }^{{ }^{*}}$ (D)

\begin{abstract}
Background: Protein-protein interactions (PPIS) are fundamental to the growth and survival of cells and serve as excellent targets to develop inhibitors of biological processes such as host-pathogen interactions and cancer cell proliferation. However, isolation of PPI inhibitors is extremely challenging. While several in vitro assays to screen for PPI inhibitors are available, they are often expensive, cumbersome, and require large amounts of purified protein. In contrast, limited in vivo assays are available to screen for small-molecule inhibitors of PPI.

Methods: We have engineered a yeast strain that is suitable for screening of small-molecule inhibitors of proteinprotein interaction using the Yeast 2-hybrid Assay. We have optimised and validated the assay using inhibitors of the p53-Mdm2 interaction and identified a hitherto unreported putative Mdm2-binding domain in p53.

Results: We report a significantly improved and thoroughly validated yeast two-hybrid ( $\mathrm{Y} 2 \mathrm{H})$ assay that can be used in a high throughput manner to screen for small-molecule PPI inhibitors. Using the p53-Mdm2 interaction to optimize the assay, we show that the p53-Mdm2 inhibitor nutlin-3 is a substrate for the yeast ATP-binding cassette (ABC) transporter Pdr5. By deleting nine ABC transporter-related genes, we generated a ABC9 $\triangle$ yeast strain that is highly permeable to small molecules. In the ABC9 $\Delta$ strain, p53-Mdm2 interaction inhibitors, like AMG232 and MI-773, completely inhibited the p53-Mdm2 interaction at nanomolar concentrations in the $\mathrm{Y} 2 \mathrm{H}$ assay. In addition, we identified a conserved segment in the core DNA-binding domain of p53 that facilitates stable interaction with Mdm2 in yeast cells and in vitro.
\end{abstract}

Conclusion: The $\mathrm{Y} 2 \mathrm{H}$ assay can be utilized for high-throughput screening of small-molecule inhibitors of PPIs and to identify domains that stabilize PPIs.

Keywords: Yeast two-hybrid assay, Protein-protein interaction inhibitors, p53-Mdm2 interaction

\footnotetext{
*Correspondence: prakasha@bii.a-star.edu.sg

'Bioinformatics Institute, 30 Biopolis Street, \#07-01, Matrix, Singapore 138671,

Singapore

Full list of author information is available at the end of the article
} 


\section{Background}

A multitude of biological processes are dependent on protein-protein interactions (PPIs). It has been estimated that approximately 350,000 types of PPIs occur in a human cell [1]. This offers innumerable opportunities to develop PPI inhibitors and thus to specifically control cellular processes. However, obtaining a small-molecule inhibitor of PPIs is not trivial. Generally, the interfaces involved in PPIs are flat and large, thereby reducing the probabilities of effective competition by a smallmolecule inhibitor [2]. However, there has been some success over the last couple of decades in obtaining small-molecule PPI inhibitors [3]. It has been observed that a few key residues in the PPI interface contribute to the bulk of the binding energy in a PPI [4] and, thus, could be targeted for inhibition by a small molecule.

In order to sample the entire conformational space for PPIs, a simple inexpensive screening assay usable in a highthroughput format is extremely desirable. Although there are in vitro assays involving methods such as isothermal titration calorimetry, surface plasmon resonance, microscale thermophoresis, enzyme-linked immunosorbent assay, fluorescence polarization, fluorescence resonance energy transfer, and the bead-based AlphaScreen assay (Perkin Elmer), they are often labour intensive, expensive, and require large amounts of purified protein [5]. Moreover, in vitro assays may identify compounds that are good PPI inhibitors but may have toxic effects or low cell permeability. Therefore, it is advantageous to have an economic, high throughput in vivo assay to screen for PPI inhibitors.

The yeast two-hybrid $(\mathrm{Y} 2 \mathrm{H})$ assay is a powerful tool to identify binary PPIs [6] by exploiting the modular nature of the yeast Gal4 transcription factor. In this assay, the DNAbinding domain and activation domain of Gal4 are fused to two proteins of interest. If the two proteins of interest physically interact, an active Gal4 transcription factor is generated, thereby driving expression of reporter genes under the control of the GAL promoter (Fig. 1a, left panel). Apart from confirming an interaction between two proteins, this assay has been pivotal in discovering novel binding proteins. The $\mathrm{Y} 2 \mathrm{H}$ assay has been used in developing binary protein interactome maps in model organisms such as yeast [7] and humans [8].

The $\mathrm{Y} 2 \mathrm{H}$ assay can also be used to identify domains and amino acid residues required for PPIs. Deletion or replacement of amino acid residues critical for PPI or treatment with small-molecule PPI inhibitors will result in loss of reporter gene activity (Fig. 1a, right panel). It is possible to have a positive selection for screening of mutations or compounds that affect PPIs. For example, by placing the $U R A 3$ gene under the GAL promoter, one could screen for mutations or PPI inhibitors that rescue the lethality of yeast cells grown on medium containing 5-fluoroorotic acid; this approach is referred to as the reverse $\mathrm{Y} 2 \mathrm{H}$ assay and was proposed 20 years ago [9, $10]$. However, there are very few reports of its use in screening of PPI inhibitors $[11,12]$. It has been acknowledged that low permeability of yeast cells to small molecules could limit the use of $\mathrm{Y} 2 \mathrm{H}$ methods to screen for PPI inhibitors [13].

To explore the use of the $\mathrm{Y} 2 \mathrm{H}$ assay to screen for inhibitors of PPIs, we chose the p53-Mdm2 interaction, for which there are several small-molecule inhibitors available. p53 is a master transcription factor that plays a key role in the regulation of cell cycle arrest, DNA damage response, senescence, and apoptosis [14]; it is mutated in more than $50 \%$ of cancers [15]. p53 is inhibited by $\mathrm{Mdm} 2$, a ubiquitin ligase that is often overexpressed in tumors [16]. By binding to the N-terminal transactivation domain of $\mathrm{p} 53, \mathrm{Mdm} 2$ inhibits its transcriptional activity, ubiquitinates and targets it for proteosomal degradation, and excludes it from the nucleus. Inhibition of the p53-Mdm2 interaction leads to activation of p53 and an increase in its tumor suppressive ability. The p53Mdm2 interaction can be attributed to three key hotspot residues (Phe19, Trp23, and Leu26) in p53 that bind to a hydrophobic pocket on the surface of the Mdm2's Nterminal domain [17] (Additional file 1: Figure S1A). Small-molecule inhibitors, such as nutlin, AMG232, and MI-773, bind to the hydrophobic pocket of Mdm2 and inhibit the p53-Mdm2 interaction by mimicking the interaction of the three hydrophobic residues [18-21] (Additional file 1: Figure S1B-D). Binding of Mdm2 to full-length p53 was observed to be approximately 10fold stronger than the $\mathrm{N}$-terminal domain of p53 (amino acid residues 1-93) [22], indicating the presence of additional domains in p53 that interact with Mdm2. Two such domains have thus far been reported; the DNAbinding domain of p53 (residues 234-286 within the conserved Boxes IV and V) has been shown to interact with the central acidic domain of Mdm2 [23] and the carboxyl-terminal regulatory domain of p53 (residues 367-393) interacts with the N-terminal domain of Mdm2 (residues 10-139) [24].

In this paper, we report on an improved version of the $\mathrm{Y} 2 \mathrm{H}$ assay that can be used to screen for inhibitors of PPI in a high throughput manner. By deleting nine genes involved in $\mathrm{ABC}$ transporter gene function and expression, we constructed a yeast strain that is highly permeable to small molecules. We show that nutlin, AMG232, and MI-773 can inhibit the p53-Mdm2 interaction in the $\mathrm{Y} 2 \mathrm{H}$ assay. We demonstrate that nutlin is a substrate for the yeast $A B C$ transporter Pdr5. In addition, we suggest that the Box II region in the core DNA-binding domain of p53 interacts with the $\mathrm{N}$-terminal domain of $\mathrm{Mdm} 2$ and makes the p53-Mdm2 interaction recalcitrant to small-molecule inhibition in the $\mathrm{Y} 2 \mathrm{H}$ assay. 

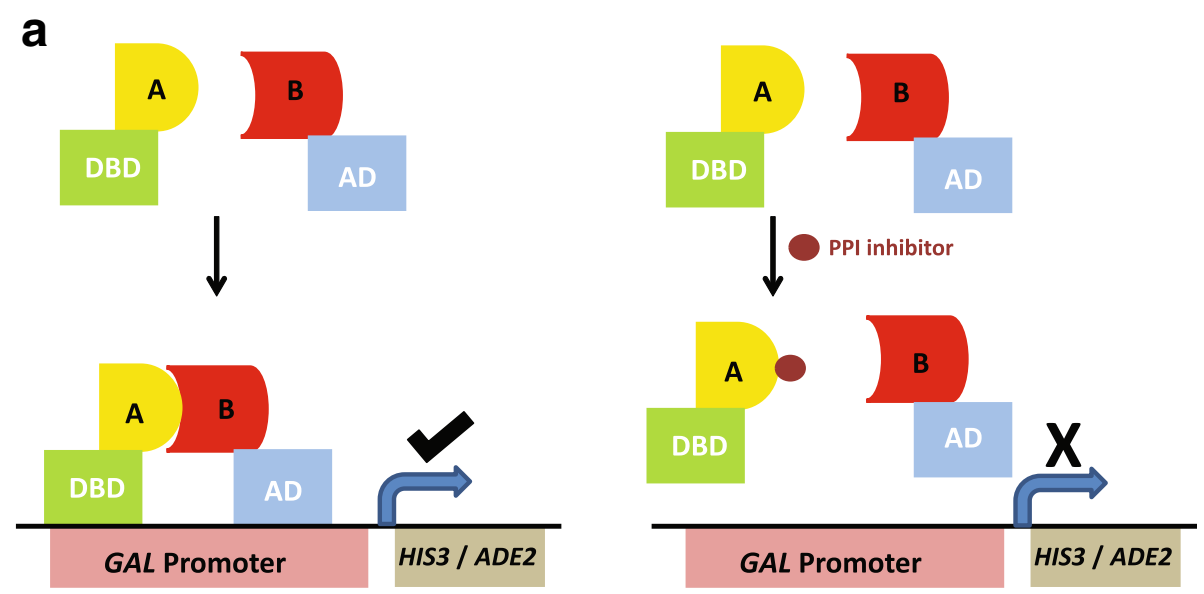

b

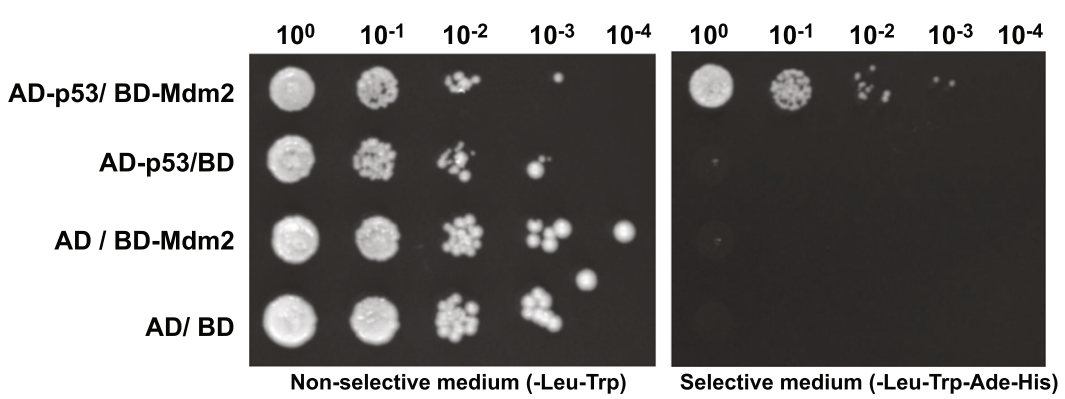

C

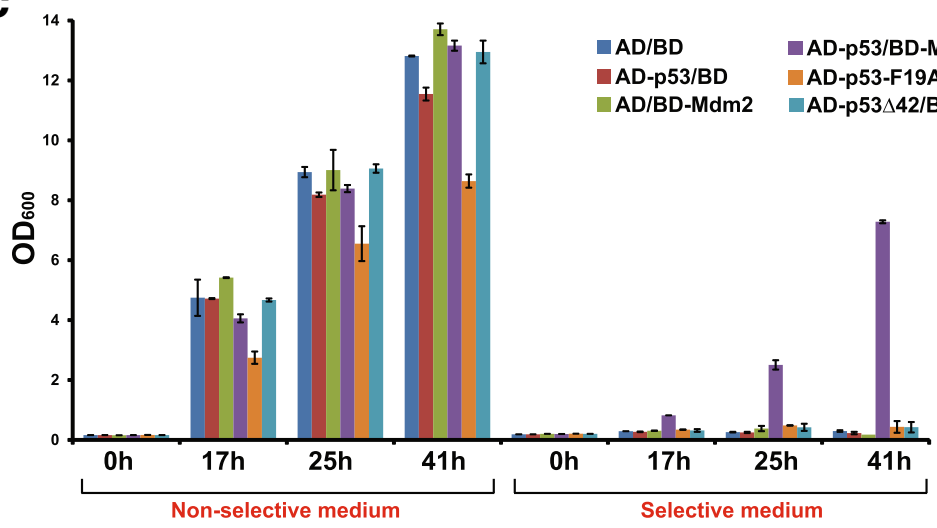

Fig. 1 p53 interacts with Mdm2 in the yeast two-hybrid $(\mathrm{Y} 2 \mathrm{H})$ assay. a Schematic showing the use of the $\mathrm{Y} 2 \mathrm{H}$ assay in identifying interacting proteins (left panel) and inhibitors of protein-protein interactions (right panel). $\mathbf{b}$ Log-phase cultures of AH109 yeast cells containing plasmids encoding either Gal4 AD-p53/Gal4 BD-Mdm2, Gal4 AD-p53/Gal4 BD, Gal4 AD/Gal4 BD-Mdm2, or Gal4 AD/Gal4 BD were washed in water and plated at different dilutions on non-selective (-Leu-Trp) and selective (-Leu-Trp-Ade-His) plates and incubated at $30^{\circ} \mathrm{C}$ for 3 days. c Overnight cultures of AH109 yeast cells containing plasmids encoding either Gal4 AD-p53/Gal4 BD-Mdm2, Gal4 AD-p53/Gal4 BD, Gal4 AD/Gal4 BD-Mdm2, Gal4 AD/Gal4 BD, Gal4 AD-p53-F19A/Gal4 BD-Mdm2, or Gal4 AD-p53( $\triangle 42) / G a l 4$ BD-Mdm2 in non-selective medium were washed in water and inoculated into selective and non-selective medium at $\mathrm{OD}_{600}=0.2$ in duplicates. For each strain, growth as measured by average $\mathrm{OD}_{600}$ of duplicate cultures is plotted against time. Ends of the vertical bar indicate the $\mathrm{OD}_{600}$ values of the duplicate cultures

\section{Results}

p53 interacts with $\mathrm{Mdm} 2$ in the $\mathrm{Y} 2 \mathrm{H}$ assay

The p53-Mdm2 interaction is of immense biomedical importance and is a focus of several screens as a target for small-molecule inhibitors [18]. As there are many smallmolecule inhibitors available, it is an ideal system to optimize the $\mathrm{Y} 2 \mathrm{H}$ assay [18]. We first established a $\mathrm{Y} 2 \mathrm{H}$ assay to detect the p53-Mdm2 interaction. We fused the full-length human p53 ( $\mathrm{Hp} 53$ ) to the Gal4 activation domain (Gal4 AD) and the full-length human Mdm2 (hMdm2) to the Gal4 DNA-binding domain (Gal4 BD), and introduced plasmids encoding them into the $\mathrm{Y} 2 \mathrm{H}$ reporter strain AH109. AH109 has HIS3 and ADE2 genes under the GAL4 promoter and an interaction between 
Gal4 AD- and Gal4 BD-fusion proteins results in growth of yeast cells in Synthetic Dropout (SD) medium lacking histidine and adenine (Fig. 1a, left panel). As expected, only cells containing both plasmids encoding Gal4 ADp53 and Gal4 DBD-Mdm2, but not the control cells, were able to grow in SD medium lacking histidine and adenine (Fig. 1b). Either deletion of the transactivation domain (1-42) or replacement of the hydrophobic triad residue phenylalanine F19 (which is involved in binding to the hydrophobic pocket of Mdm2) with alanine completely abolished growth of Gal4 AD-p53/Gal4 BD-Mdm2expressing cells in selective medium, indicating the specificity of the interaction (Fig. 1c).

Nutlin does not inhibit the p53-Mdm2 interaction in wildtype and $p d r 1 \Delta p d r 3 \Delta p d r 5 \Delta$ yeast cells in the $\mathrm{Y} 2 \mathrm{H}$ assay Nutlin is a cis-imidazoline analogue that binds to the p53-binding site in Mdm2 (Additional file 1: Figure S1) and inhibits the p53-Mdm2 interaction [19]. Thus, we tested whether nutlin inhibits the p53-Mdm2 interaction in the $\mathrm{Y} 2 \mathrm{H}$ assay. Surprisingly, nutlin had no effect on the growth of $\mathrm{p} 53-\mathrm{Mdm} 2$-expressing cells in selective medium (Additional file 2: Figure S2A). We considered the possibility that nutlin was being expelled from yeast cells by $\mathrm{ABC}$ transporter-mediated efflux. We deleted three genes, PDR1, PDR3, and PDR5, which have been reported to restrict permeability of yeast cells to small molecules [25], to construct the $p d r 1 \Delta p d r 3 \Delta p d r 5 \Delta$ strain (referred to as $\mathrm{ABC} 3 \Delta$ for the remainder of this paper). Pdr1 and Pdr3 are transcription factors for the pleiotropic drug response and $\operatorname{Pdr} 5$ is an $\mathrm{ABC}$ transporter. In the $\mathrm{ABC} 3 \Delta$ strain, nutlin inhibited p53-Mdm2 cell growth at $200 \mu \mathrm{M}$. However, nutlin also inhibited the growth of control cells expressing Csm1/Dsn1, a pair of interacting yeast kinetochore proteins [23, 26], indicating that the growth inhibition of yeast cells by nutlin was not specific (Additional file 2: Figure S2B).

\section{Nutlin inhibits the interaction of p53 (1-52) with Mdm2 in pdr1 $\Delta$ pdr3 $\Delta$ pdr5 $\Delta$ cells}

We then considered the nature of the p53-Mdm2 interaction. While the transactivation domain of $\mathrm{p} 53$ interacts with the $\mathrm{N}$-terminal domain of $\mathrm{Mdm} 2$, the core domain and C-terminal regulatory domain of p53 also respectively interact with the acidic- and $\mathrm{N}$ terminal domains of $\mathrm{Mdm} 2$ [23, 24]. To eliminate the possible effect of the core domain and C-terminal tail, we expressed p53 (1-52) fused to Gal4 AD and examined the effect of nutlin on its interaction with Gal4 DBD-Mdm 2 in ABC3 $\Delta$ cells. The interaction of p53 (1-52) with Mdm2 was completely inhibited at 125 $\mu \mathrm{M}$ nutlin (Fig. 2a). In contrast, the interaction of fulllength p53 with Mdm2 was only partially inhibited (29\%), even at $150 \mu \mathrm{M}$ nutlin. Cells expressing Csm1/
Dsn1 in selective medium were unaffected by the addition of $150 \mu \mathrm{M}$ nutlin, indicating that the inhibition of PPIs by nutlin was specific (Fig. 2a). Moreover, nutlin did not affect the growth of cells expressing p53 (1-52) and Mdm2 in non-selective medium (Additional file 2: Figure S2C). Nutlin did not inhibit the growth of wild-type cells expressing p53 (1-52) and $\mathrm{Mdm} 2$, confirming that $\mathrm{ABC}$ transporters drive nutlin out of yeast cells (Fig. 2a).

As expected, the interaction of p53 (1-52) with the N-terminal domain of Mdm2 (1-125) was also sensitive to nutlin (Fig. 2b and Additional file 3: Figure S3). To confirm that nutlin inhibits the growth of p53 (1-52)/Mdm 2 cells by binding to the N-terminal domain of Mdm2, we introduced the M62A mutation in Mdm2 (1-125), which blocks its interaction with nutlin but retains its interaction with p53 [27]. Crucially, nutlin had no effect on the growth of cells expressing p53 (1-52)/Mdm2 (1-125)-M62A (Fig. 2b and Additional file 3: Figure S3), confirming that nutlin blocks the p53-Mdm2 interaction by binding to $\mathrm{Mdm} 2$.

\section{Nutlin is a substrate for the $A B C$ transporter Pdr5}

Inhibition of the p53 (1-52)/Mdm2 interaction by nutlin in ABC3 $\Delta$ cells but not in wild-type cells (Fig. 2a) suggested that Pdr1, Pdr3, and Pdr5 proteins, either singly or in combination, mediate nutlin efflux from yeast cells. To test which of Pdr1, Pdr3, and Pdr5 proteins contributes to nutlin efflux, we tested the effect of nutlin on the p53 (1-52)/ $\mathrm{Mdm} 2$ interaction in single mutants. Interestingly, nutlin inhibited the p53 (1-52)/Mdm2 interaction only in pdr5 $\Delta$ cells but not in $p d r 1 \Delta$ and $p d r 3 \Delta$ cells (Additional file 4: Figure S4), indicating that Pdr5 causes efflux of nutlin from yeast cells.

The Saccharomyces cerevisiae proteome is predicted to have $22 \mathrm{ABC}$ transporters [28]. Although only a few of these transporters have been extensively studied, they are known to exhibit broad specificity [28]. To evaluate the specificity of Pdr5 in nutlin efflux, we deleted six genes encoding ABC transporters, namely PDR15, PDR11, PDR10, $Y C F 1, S N Q 2$, and YOR1, and tested the effect of nutlin on the p53-Mdm2 interaction in the mutants. Strikingly, the p53-Mdm2 interaction was sensitive to nutlin only in pdr5 $\Delta$ cells but not in the other eight mutants (Fig. 3a, Additional file 5: Figure S5A and Additional file 6: Figure S6). For an unrelated drug, rapamycin, pdr11 $\Delta$ and yor $1 \Delta$ were most sensitive (Additional file 5: Figure S5B), confirming that $\mathrm{ABC}$ transporters have distinct substrate specificities. Nutlin sensitivity of pdr5 $\Delta$ cells expressing p53 (1$52) / \mathrm{Mdm} 2$ was rescued by introduction of a $2-\mu$ plasmid encoding Pdr5 under the $A D H 1$ promoter (Fig. $3 \mathrm{~b}$ ). These results indicate that nutlin is a substrate of Pdr5.

To use the $\mathrm{Y} 2 \mathrm{H}$ assay for screening of PPI inhibitors, it is desirable to have a yeast strain that is permeable to a 


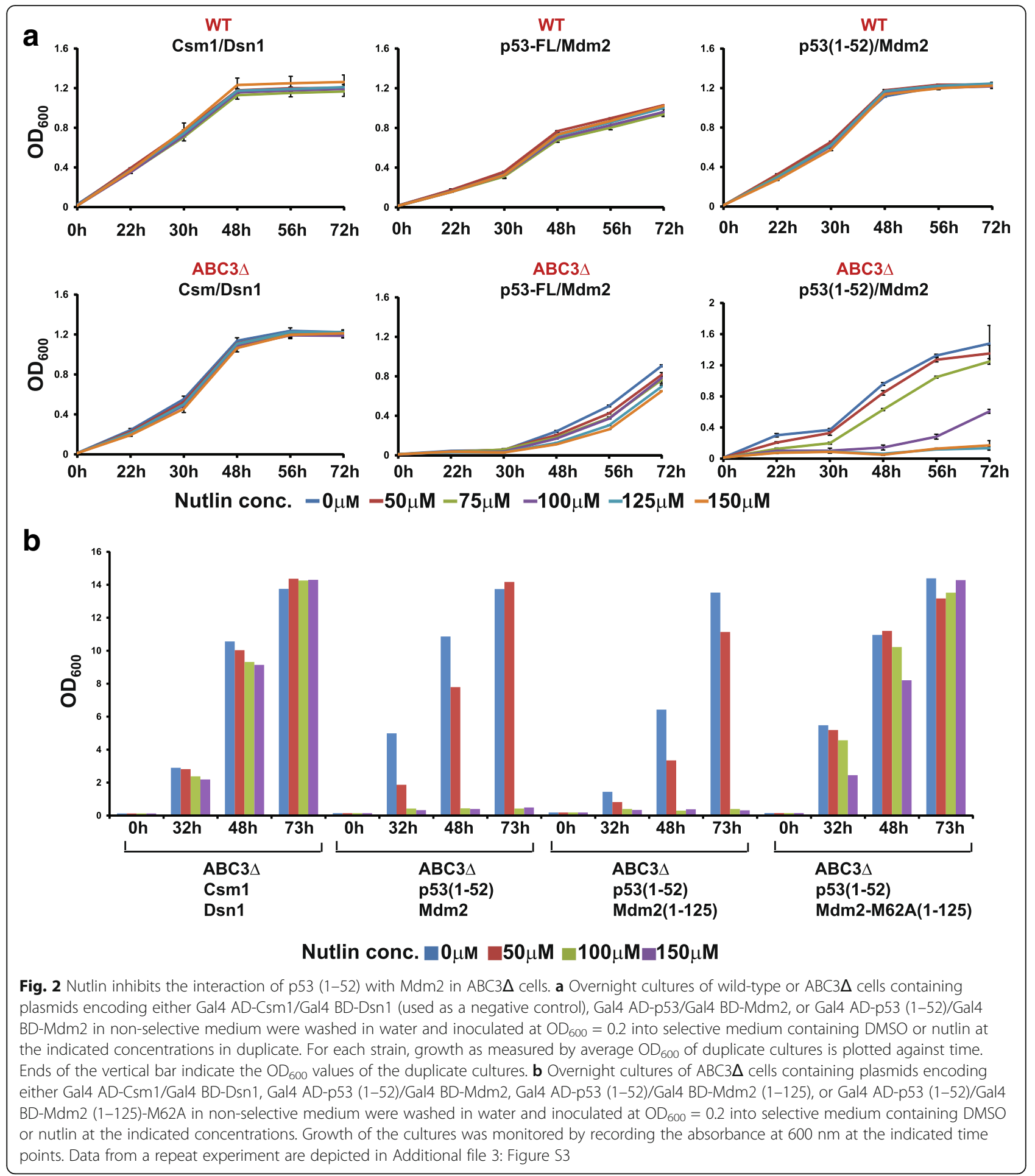

diverse collection of compounds. As $\mathrm{ABC}$ transporters have differing substrate specificities, we constructed a strain that lacks several $\mathrm{ABC}$ transporter genes. A previous study showed that a yeast strain lacking seven $A B C$ transporter genes (PDR5, PDR15, PDR11, PDR10, YCF1, SNQ2, and $Y O R 1)$ and one gene encoding a transcription factor for $\mathrm{ABC}$ transporters (PDR3) was viable [29]. We generated the $A B C 9 \triangle$ strain, a derivative of the $\mathrm{Y} 2 \mathrm{H}$ strain AH109, which contains the abovementioned eight gene deletions together with deletion of $P D R 1$. The ABC9 $\triangle$ strain 


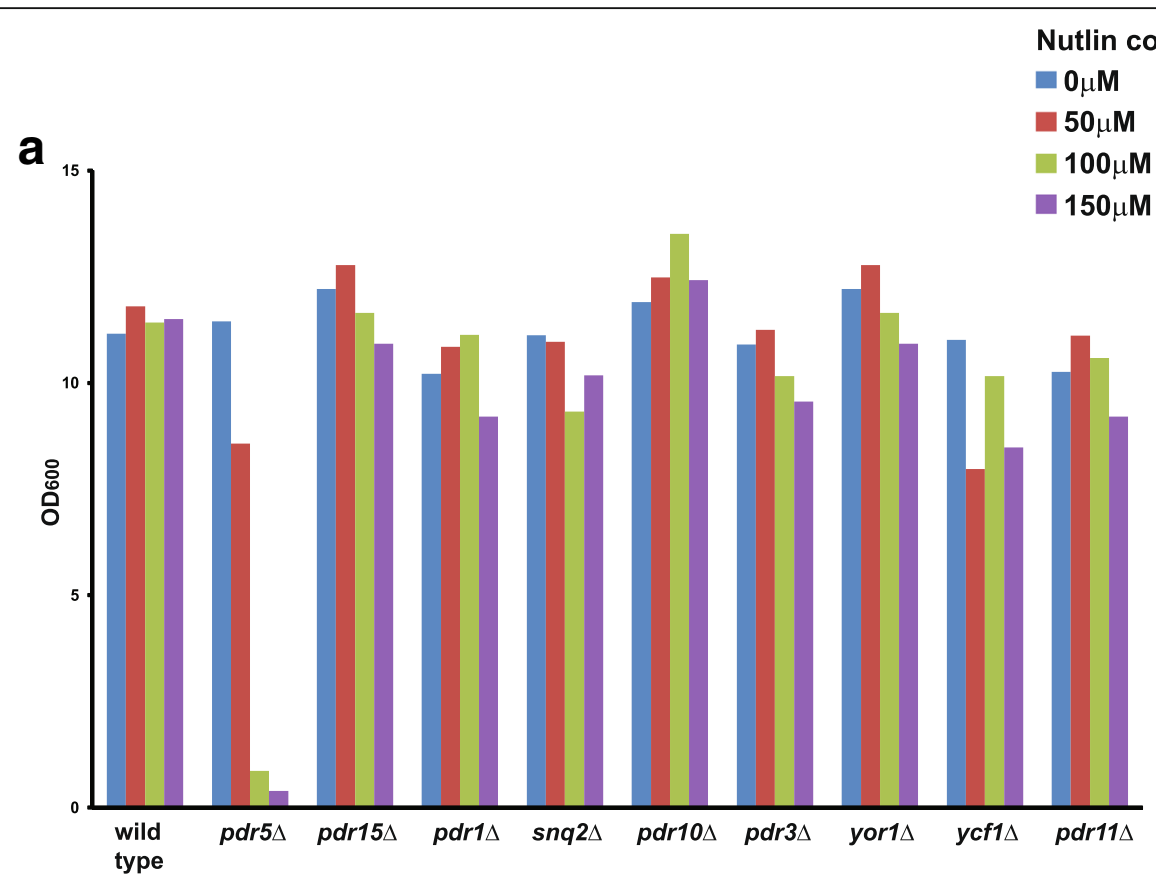

b

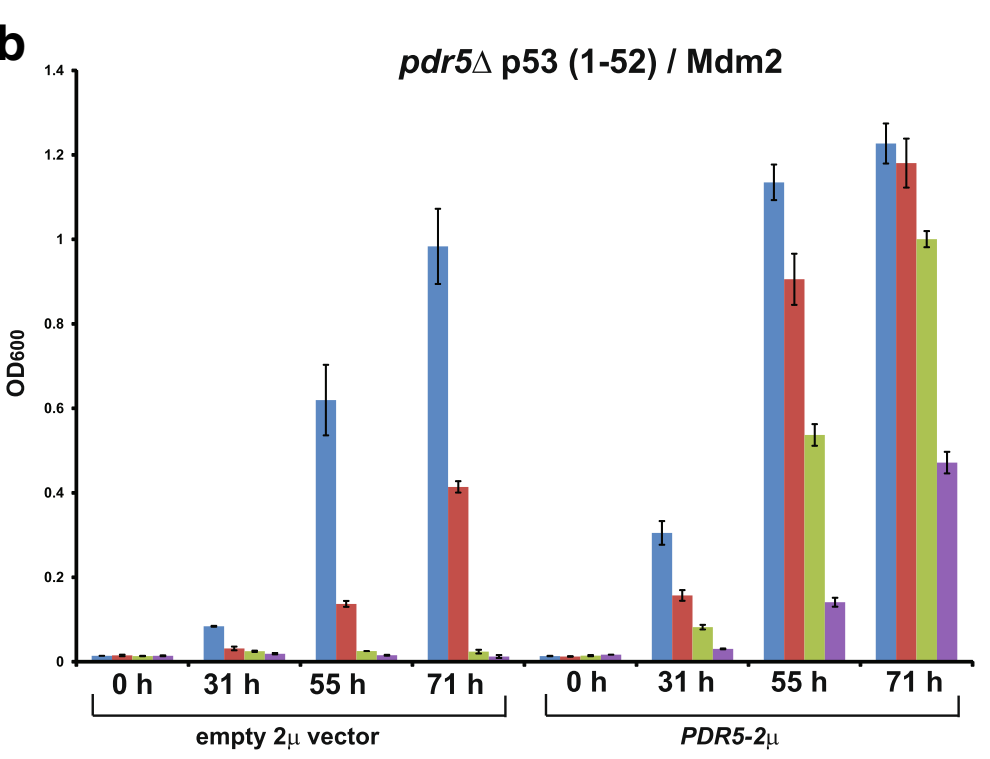

Nutlin conc.

- $0 \mu \mathrm{M}$

$\square 100 \mu \mathrm{M}$

$150 \mu \mathrm{M}$

$\square 200 \mu M$

Fig. 3 Nutlin is a substrate for the ABC transporter Pdr5. a Overnight cultures of either wild-type strain or the indicated deletion strains ( $p d r 5 \Delta$, pdr15 $\Delta$, pdr1 $\Delta$, etc.) containing plasmids encoding Gal4 AD-p53 (1-52)/Gal4 BD-Mdm2 were washed in water and inoculated at $\mathrm{OD}_{600}=0.2$ into selective medium containing DMSO or nutlin at the indicated concentrations. Growth of the cultures was monitored by recording the absorbance at $600 \mathrm{~nm}$ after $0,24,44$, and $68 \mathrm{~h}$ following inoculation. Growth at $68 \mathrm{~h}$ is indicated in the plot. Additional file 5: Figure S5A contains the complete dataset and Additional file 6: Figure $\mathrm{S} 6$ contains data from a repeat experiment. b Overnight cultures of $p d r 5 \Delta$ cells containing plasmids encoding Gal4 AD-p53 (1-52)/Gal4 BD-Mdm2 and Pdr5 or Gal4 AD-p53 (1-52)/Gal4 BD-Mdm2 alone were washed in water and inoculated at $\mathrm{OD}_{600}=0.2$ into selective medium containing DMSO or nutlin at the indicated concentrations in duplicate. For both strains, growth as measured by average $\mathrm{OD}_{600}$ of duplicate cultures is plotted against time. Ends of the vertical bar indicate the $\mathrm{OD}_{600}$ values of the duplicate cultures

was hypersensitive to doxorubicin, myriocin, cycloheximide, and nutlin in comparison to the wild-type strain, indicating that it is permeable to several compounds (Fig. 4a). This is consistent with earlier studies showing that $\mathrm{ABC}$ transporter mutants in yeast are sensitive to doxorubicin, myriocin, and cycloheximide [30, 31]. In the $\mathrm{ABC} 9 \Delta$ strain, the p53 (1-52)/Mdm2 interaction was sensitive to nutlin at $10 \mu \mathrm{M}$ in comparison to $100 \mu \mathrm{M}$ in the $\mathrm{ABC} 3 \Delta$ strain (Figs. 4b and Additional file 7: Figure S7). This value $(10 \mu \mathrm{M})$ fits closely with the potency of nutlin 

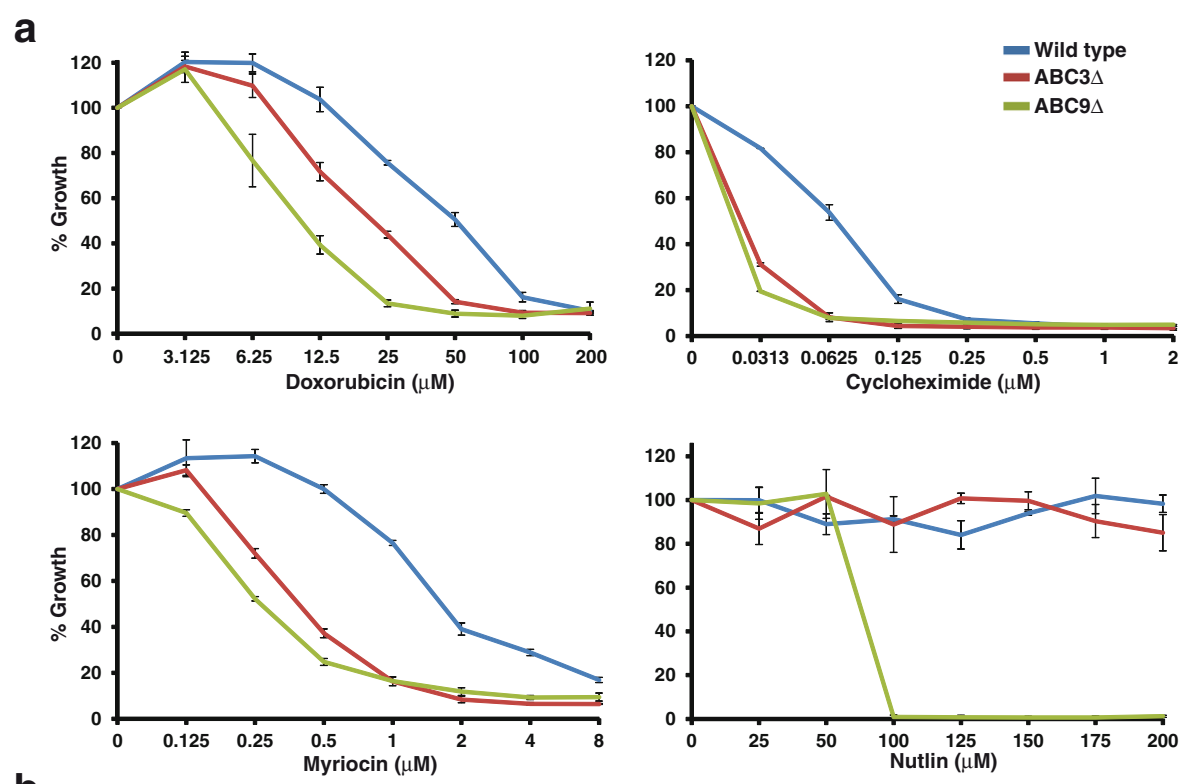

b

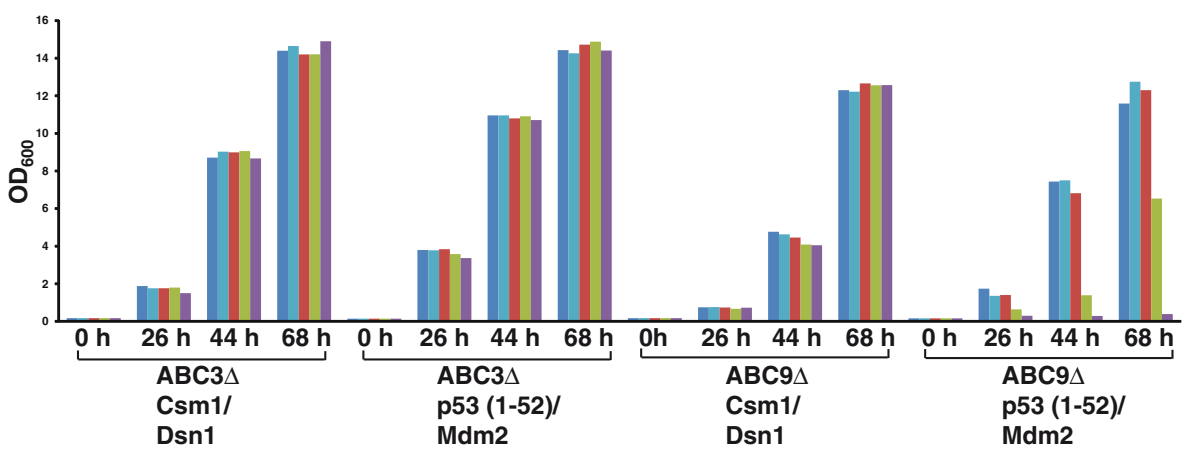

Nutlin conc. $\square 0 \mu \mathrm{M} \square 500 \mathrm{nM} \square 1 \mu \mathrm{M} \square 5 \mu \mathrm{M} \square 10 \mu \mathrm{M}$

Fig. 4 The $A B C 9 \Delta$ strain has increased permeability to several compounds. a Overnight cultures of either wild-type, $A B C 3 \Delta$ ( $p d r 1 \Delta p d r 3 \Delta p d r 5 \Delta$ ), or $\mathrm{ABC} \Delta \Delta$ ( $p d r 1 \Delta p d r 3 \Delta p d r 5 \Delta$ snq $2 \Delta$ pdr10 $\Delta$ pdr11 $\Delta$ pdr15 $\Delta$ yor $1 \Delta$ ycfi $\Delta$ ) cells in rich medium (YEPD) were washed in water and inoculated at $\mathrm{OD}_{600}=0.0625$ into YEPD containing either DMSO, doxorubicin, cycloheximide, myriocin, or nutlin at the indicated concentrations in duplicate. For each strain, growth as measured by average $\mathrm{OD}_{600}$ of duplicate cultures after $40 \mathrm{~h}$ following inoculation was recorded. Percentage inhibition at different concentrations was calculated by normalizing with respect to the growth in DMSO-treated cultures. Ends of the vertical bar indicate values of percentage inhibition for the duplicate cultures. $\mathbf{b}$ Overnight cultures of $A B C 3 \Delta$ or $A B C 9 \Delta$ cells containing plasmids encoding either Gal4 AD-Csm1/Gal4 BD-Dsn1 or Gal4 AD-p53 (1-52)/Gal4 BD-Mdm2 in non-selective medium were washed in water and inoculated into selective medium at $\mathrm{OD}_{600}=0.0625$ Growth of the cultures was monitored by recording the absorbance at $600 \mathrm{~nm}$ after $0,26,44$, and $68 \mathrm{~h}$ following inoculation. Data from a repeat experiment are shown in Additional file 7: Figure S7

in mammalian cells, indicating that the $\mathrm{ABC} 9 \Delta$ strain is an excellent system for small-molecule PPI inhibitor screens $[32,33]$.

\section{AMG232 and MI-773 inhibit the p53 (1-52)/Mdm2 interaction in the $\mathrm{Y} 2 \mathrm{H}$ assay}

We then tested whether the $\mathrm{Y} 2 \mathrm{H}$ assay for the p53 (1-52)/Mdm2 interaction optimized for nutlin was extendable to other p53-Mdm2 interaction inhibitors, namely AMG232 and MI-773. Like nutlin, AMG232 and MI-773 bind to the p53-binding site in Mdm2 and block the p53-Mdm2 interaction (Additional file 1: Figure S1). Both AMG232 and MI-773 abolished the p53 (1-52)/ $\mathrm{Mdm} 2$ interaction in wild-type, $\mathrm{ABC} 3 \Delta$, and $\mathrm{ABC} 9 \Delta$ strains (Fig. 5a, b). However, the p53 (1-52)/Mdm2 interaction was approximately 25 -fold more sensitive to MI-773 and AMG232 in the ABC9 $\Delta$ strain in comparison to the wild-type strain (Fig. 5a, b). 


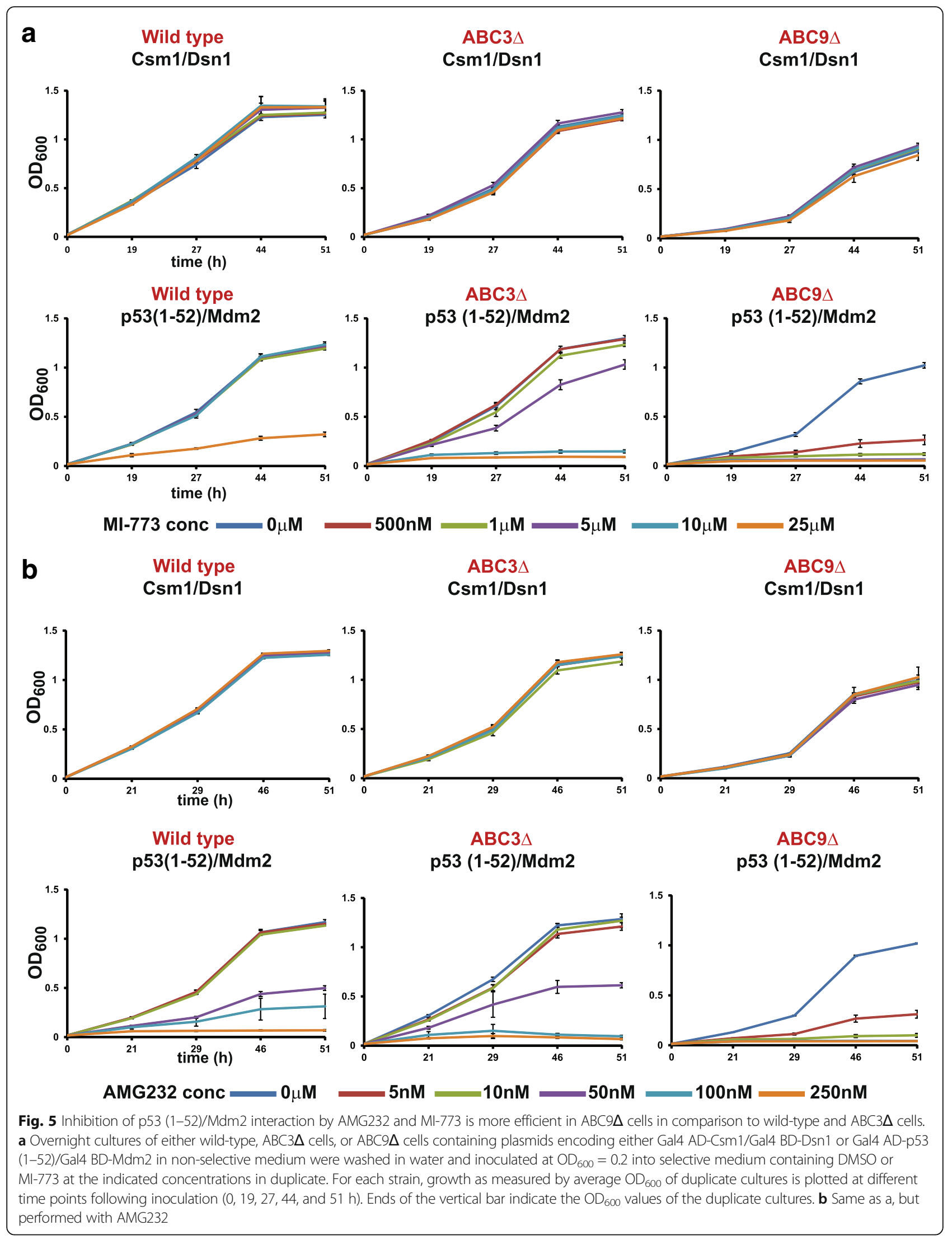


The full-length $\mathrm{p} 53 / \mathrm{Mdm} 2$ interaction is more resistant to small-molecule inhibitors in comparison to the p53 (1-52)/ Mdm2 interaction

As the p53 (1-52)/Mdm2 interaction in the ABC9 $\triangle$ strain was more sensitive to small-molecule inhibitors in comparison to wild-type and $\mathrm{ABC} 3 \Delta$ strains (Fig. 5), we tested whether the full-length $\mathrm{p} 53 / \mathrm{Mdm} 2$ interaction was sensitive to small-molecule inhibitors in the $\mathrm{ABC} 9 \Delta$ strain. Nutlin, MI-773, and AMG232 inhibited the interaction of full-length p53 with Mdm2 by approximately $70-75 \%$ (Fig. 6). Inhibition of the p53-FL/Mdm2 interaction by nutlin in the $\mathrm{ABC} 9 \Delta$ strain was approximately 2 - to 3-fold better than that achieved in the $A B C 3 \triangle$ strain (Fig. 2a). In contrast, the p53 (1-52)/Mdm2 interaction was inhibited by approximately $90-95 \%$ by the three inhibitors (Fig. 6). Taken together, our results
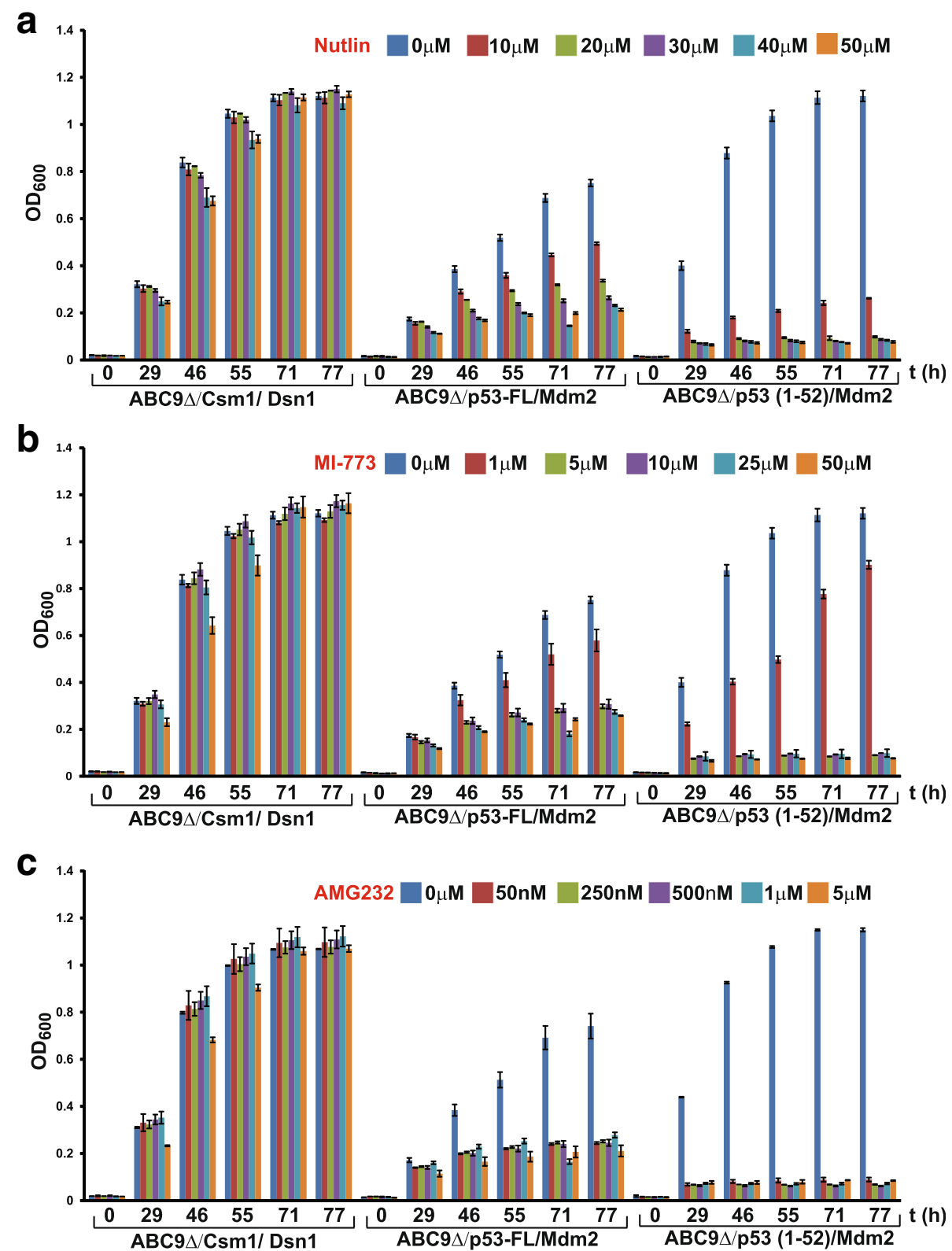

Fig. 6 The full-length $\mathrm{p53/Mdm2}$ interaction is more resistant to small-molecule inhibitors in comparison to the p53 (1-52)/Mdm2 interaction. a Overnight cultures of ABC9 $\Delta$ cells containing plasmids encoding either Gal4 AD-Csm1/Gal4 BD-Dsn1, Gal4 AD-p53FL/Gal4 BD-Mdm2, or Gal4 AD-p53 (1-52)/Gal4 BD-Mdm2 in non-selective medium were washed in water and inoculated at $\mathrm{OD}_{600}=0.2$ into selective medium containing DMSO or nutlin at the indicated concentrations in duplicate. For each strain, growth as measured by average $\mathrm{OD}_{600}$ value of duplicate cultures is plotted at different time points following inoculation $(0,29,46,55,71$, and $77 \mathrm{~h})$. Ends of the vertical bar indicate the $\mathrm{OD}_{600}$ values of the duplicate cultures. b, c Same as in a, but performed with MI-773 and AMG232, respectively 
indicate that the interaction of full-length p53 with $\mathrm{Mdm} 2$ is more resistant to small-molecule inhibitors in comparison to the p53 (1-52)/Mdm2 interaction.

\section{Highly conserved Box II in the DNA-binding domain of p53 strengthens the p53-Mdm2 interaction}

To rationalize our observation that nutlin, AMG232, and MI-773 inhibited the p53 (1-52)/Mdm2 interaction much more efficiently than the full-length p53/Mdm2 interaction in the $\mathrm{Y} 2 \mathrm{H}$ assay we hypothesized that there are additional domains in p53 besides the transactivation domain that interact with Mdm2. To map the region of p53 that contributes to nutlin resistance of the p53Mdm2 interaction, we made a series of deletions of fulllength p53 and assayed the nutlin sensitivity of their interaction with Mdm2. The results are summarized in Fig. 7a. Deletion of the C-terminal tail or the DNAbinding domain Box V of p53, which have been implicated in the interaction with $\mathrm{Mdm} 2$, had no effect on nutlin sensitivity. Interestingly, p53 (1-160), which has the first 60 residues of the DNA-binding domain, was resistant to nutlin and p53 (1-143) was partially resistant to nutlin. The interaction of p53 (1-160) with Mdm2 was abolished by the F19A mutation and by deletion of the transactivation domain (Additional file 8: Figure S8). These results indicate that, although the primary interaction of p53 (1-160) with $\mathrm{Mdm} 2$ is via the transactivation domain, the DNA-binding domain interacts with Mdm2 and stabilizes the interaction.

To map the domain of Mdm2 that is required for increased nutlin resistance of its interaction with fulllength p53, we expressed the minimal Mdm2 (25-109) required for interaction with $\mathrm{p} 53$. The interaction of Mdm2 (25-109) with p53 (1-160) and p53 (full length) was also resistant to nutlin. To test whether the decreased sensitivity of p53-Mdm2 interaction was due to increased p53 levels, we compared the protein amounts of strains expressing different p53 variants by western blotting (Additional file 9: Figure S9). Full-length p53 was expressed to a lower extent in comparison to the other p53 variants, ruling out this possibility.

To directly compare the strength of interaction between Mdm2 and different variants of p53, we used an in vitro binding assay. We prepared HA-tagged Mdm2, full-length p53, p53 (1-52), and p53 (1-160) by in vitro translation. We then mixed HA-Mdm2 with the p53 variants and assayed the interaction by coimmunoprecipitation. Full-length p53 bound specifically to Mdm2 (Fig. 7b). Interestingly, p53 (1-160) bound to Mdm2 approximately 10 -fold more efficiently than p53 (1-52). These results are consistent with our $\mathrm{Y} 2 \mathrm{H}$ results, which show that the interaction of p53 (1-52) with Mdm2 is more sensitive to small-molecule inhibitors compared to the p53 (1-160)/full-length p53 and Mdm2 interactions. We also tested the ability of p53 (1-116) and p53 (1-143) to bind to Mdm2 in the in vitro binding assay. Only p53 (1-143), but not p53 (1-116), bound to Mdm2 (Fig. 7b). Comparable amounts of Mdm2-HA were immunoprecipitated in all the binding reactions (Fig. 7c). These results are in excellent agreement with our $\mathrm{Y} 2 \mathrm{H}$ assay results.

To identify residues involved in the additional p53$\mathrm{Mdm} 2$ interaction, we examined the conserved residues around the region (116-143) of p53 that is minimally required for nutlin resistance in the $\mathrm{Y} 2 \mathrm{H}$ assay and stable interaction with $\mathrm{Mdm} 2$ in the in vitro binding assay (Fig. 8a). We split the conserved regions into three segments (Seg1-3). Interestingly, these segments lie very close to DNA in the p53-DNA co-crystal structure [34] (Additional file 10: Figure S10). We replaced the conserved residues in each segment with alanine. Mutations in segments 1 and 2, but not 3, rendered the p53 (1-143)/Mdm2 interaction nutlin sensitive (Fig. 8b). We further replaced the subset of residues in Segments 1 and 2 with alanine and determined their effect on the nutlin sensitivity of the p53 (1-143)/Mdm2 interaction. F113A (Seg1AA), G117A T118A (Seg1BA), and K120A S121A V122A (Seg1CA) mutations in Segment 1 conferred nutlin sensitivity (Additional file 11: Figure S11A). However, mutations T125A Y126A S127A (Seg2BA) and L130A N131A K132A (Seg2CA) in Segment 2 had no effect on nutlin resistance (Additional file 11: Figure S11A). The T123A C124A T125A Y126A S127A (Seg2AA) mutation in Segment 2 abolished growth in selective medium (Additional file 11: Figure S11A). We confirmed that the wild-type and mutant p53 (1-143) proteins were expressed at comparable levels by western blotting (Additional file 11: Figure S11B). As multiple mutations in Segment 1 conferred nutlin sensitivity, we focused on the Seg1 mutant for further analysis.

We tested whether the Seg1A mutant was defective in interaction with $\mathrm{Mdm} 2$ in the in vitro binding assay. Crucially, only wild-type p53 (1-143), but not its Seg1A mutant variant, interacted with $\mathrm{Mdm} 2$ in vitro (Fig. 8c). However, the Seg1A mutation did not have an effect on the full-length p53-Mdm2 interaction (Fig. 8c). Comparable amounts of Mdm2-HA were immunoprecipitated in all the binding reactions (Additional file 12: Figure S12). In agreement with this result, the Seg1A mutation also did not have any effect on the p53-FL/Mdm2 interaction in the $\mathrm{Y} 2 \mathrm{H}$ assay (Additional file 13: Figure S13).

We suggest that additional domains in p53 that interact with Mdm2 mask the effect of Seg1A mutation on the p53-Mdm2 interaction. This is consistent with our observation that full-length p53 binds more strongly to Mdm2 in comparison to p53 (1-143) (Figs. 7b and 8c). Although the physiological significance of the Seg1/ $\mathrm{Mdm} 2$ interaction remains to be clarified, there was an 


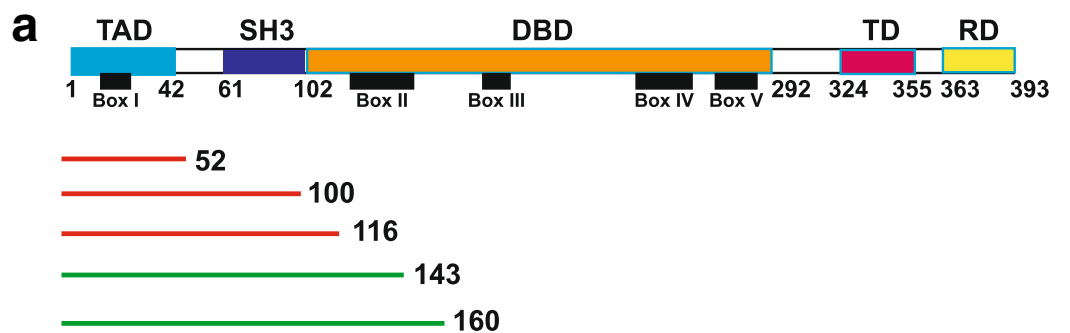

306

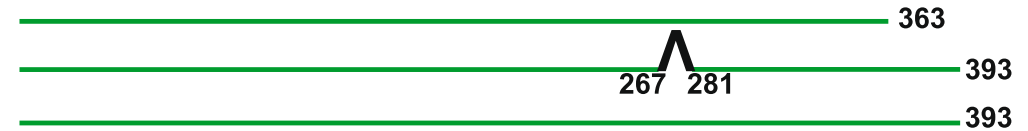

b
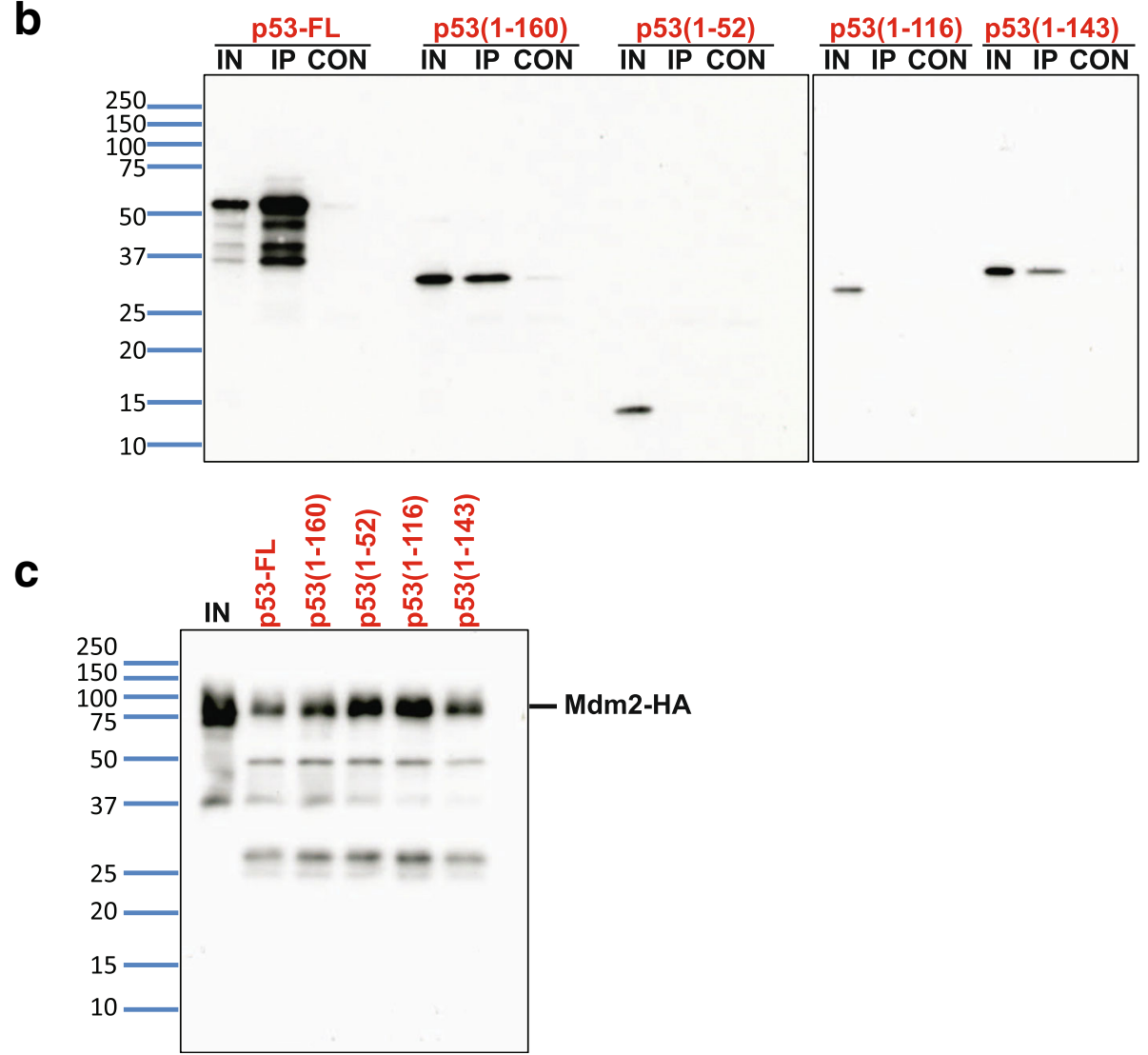

Fig. 7 The Box II region of p53's DNA-binding domain (DBD) promotes interaction with Mdm2. a Analyses of domains in p53 required for nutlin-resistant interaction with Mdm2 is summarized here. Locations of the transactivation domain (TAD), the proline rich SH3 domain, the DBD, the tetramerization domain (TD), the regulatory domain (RD), and the highly conserved Boxes (I to $V$ are indicated in the primary structure of human p53. The p53 constructs indicated by red and green lines are nutlin sensitive and nutlin resistant, respectively, in the Y2H assay with Mdm2. b Mdm2-HA, full-length p53 (p53-FL), p53 (1-160), p53 (1-52), p53 (1-116), and p53 (1-143) proteins were synthesized by in vitro translation. Binding assay was performed by incubating Mdm2-HA with the different p53 variants, followed by immunoprecipitation of Mdm2-HA using an anti-HA antibody and measuring the amount of co-immunoprecipitated p53 by western blotting analysis using anti-p53 (DO-1) antibody. Input (IN), immunoprecipitated proteins (IP), and eluate from control IP performed without Mdm2-HA (CON) were loaded on SDS-PAGE gels. c Amount of Mdm2-HA immunoprecipitated in b was analyzed by western blotting using an anti-HA antibody. IN is diluted five-fold in comparison to the IP samples for the different binding reactions (indicated in red)

excellent correspondence between sensitivity of the p53$\mathrm{Mdm} 2$ interaction to small-molecule inhibitors in the $\mathrm{Y} 2 \mathrm{H}$ assay and their stable association in the in vitro binding assay.

\section{A two-phased strategy for screening of PPI inhibitors using the $\mathrm{Y} 2 \mathrm{H}$ assay}

The enhanced permeability of the ABC9 $\Delta$ strain coupled with economic aspects of high-throughput, growth- 
a

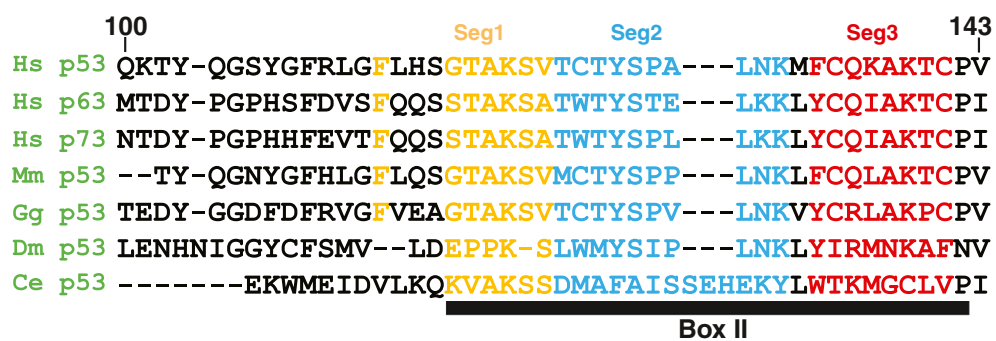

b

Nutlin conc. $₫ 0 \mu \mathrm{M} \backsim 50 \mu \mathrm{M} \backsim 100 \mu \mathrm{M} \backsim 125 \mu \mathrm{M} \backsim 150 \mu \mathrm{M}$

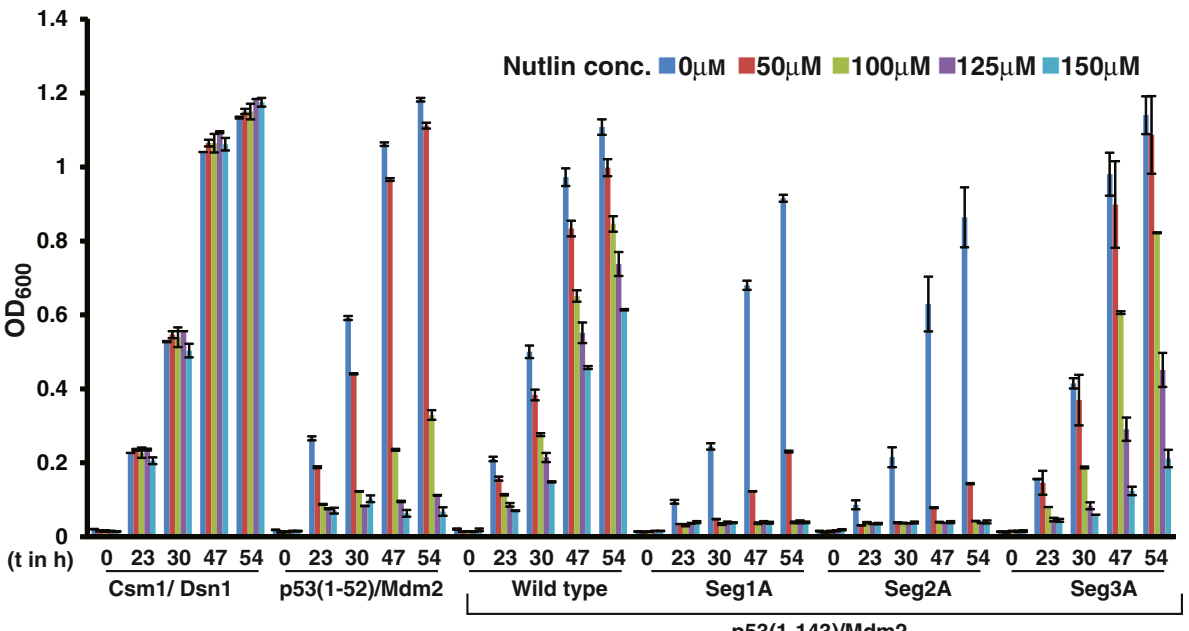

C

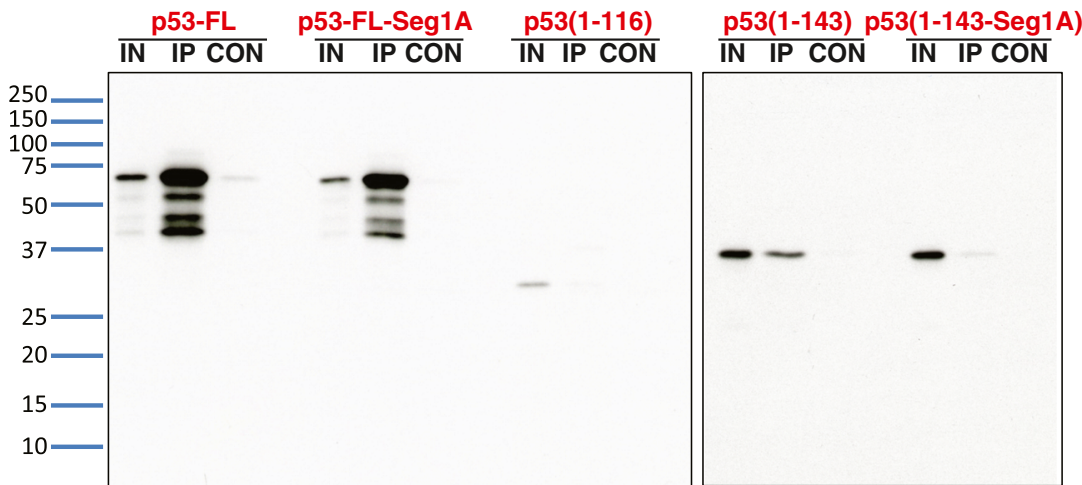

Fig. 8 The conserved Segment 1 in p53's DNA-binding domain promotes interaction with Mdm2. a Alignment of amino acid residues 100-143 of Homo sapiens p53 with the homologous amino acid sequences of Homo sapiens p63, Homo sapiens p73, Mus musculus p53, Gallus gallus p53, Drosophila melanogaster p53, and Caenorhabditis elegans p53. Conserved Segments 1, 2, and 3 are indicated in yellow, cyan, and red, respectively. Location of the conserved Box II is indicated by the black rectangle. $\mathbf{b}$ Overnight cultures of $A B C 3 \Delta$ cells containing plasmids encoding either Gal4 AD-Csm1/Gal4 BD-Dsn1, Gal4 AD-p53 (1-52)/Gal4 BD-Mdm2, Gal4 AD-p53 (1-143)/Gal4 BD-Mdm2, Gal4 AD-p53 (1-143-Seg1A)/Gal4 BD-Mdm2, Gal4 AD-p53 (1-143-Seg2A)/Gal4 BD-Mdm2, or Gal4 AD-p53 (1-143-Seg3A)/Gal4 BD-Mdm2 in non-selective medium were washed in water and inoculated at $\mathrm{OD}_{600}=0.2$ into selective medium containing DMSO or nutlin at the indicated concentrations. Growth of the cultures was monitored by recording the absorbance at $600 \mathrm{~nm}$ after $0,23,30,47$, and $54 \mathrm{~h}$ following inoculation. Ends of the vertical bar indicate the $\mathrm{OD}_{600}$ values of the duplicate cultures. c Mdm2-HA, full-length p53 (p53-FL), full-length p53 with Seg1A mutation (p53-FL-Seg1A), p53 (1-116), p53 (1-143), and p53 (1-143-Seg1A) were synthesized by in vitro translation. The binding assay was performed by incubating Mdm2-HA with the different p53 variants, followed by immunoprecipitation of Mdm2-HA using an anti-HA antibody and measuring the amount of co-immunoprecipitated p53 by western blotting analysis using anti-p53 (DO-1) antibody. Input (IN), immunoprecipitated proteins (IP), and eluate from control IP performed without Mdm2-HA (CON) were loaded on SDS-PAGE gels 
based assays makes the $\mathrm{Y} 2 \mathrm{H}$ assay suitable for screening of PPI inhibitors. In Fig. 9, we depict a methodological flowchart using a two-phased $\mathrm{Y} 2 \mathrm{H}$ assay to screen for inhibitors of PPI. In the first phase, a Y2H assay for PPIs of interest is established and validated by mutagenesis. Based on our experience with the p53-Mdm2 interaction, it would be prudent to identify domains minimally required for PPIs by truncations as weak secondary PPIs affect the sensitivity to small-molecule inhibition. Experiments for the first phase can be performed in the standard wild-type yeast strain for two-hybrid analysis. In the second phase, the plasmids encoding the minimal set of specifically interacting protein domains are introduced into the $A B C 9 \Delta$ strain, which is permeable to small molecules. Transformants pre-grown in nonselective medium are split into two and transferred into selective and non-selective media containing smallmolecule compounds in a microtiter plate. Compounds toxic to yeast cells will inhibit growth in both selective and non-selective media. However, compounds that

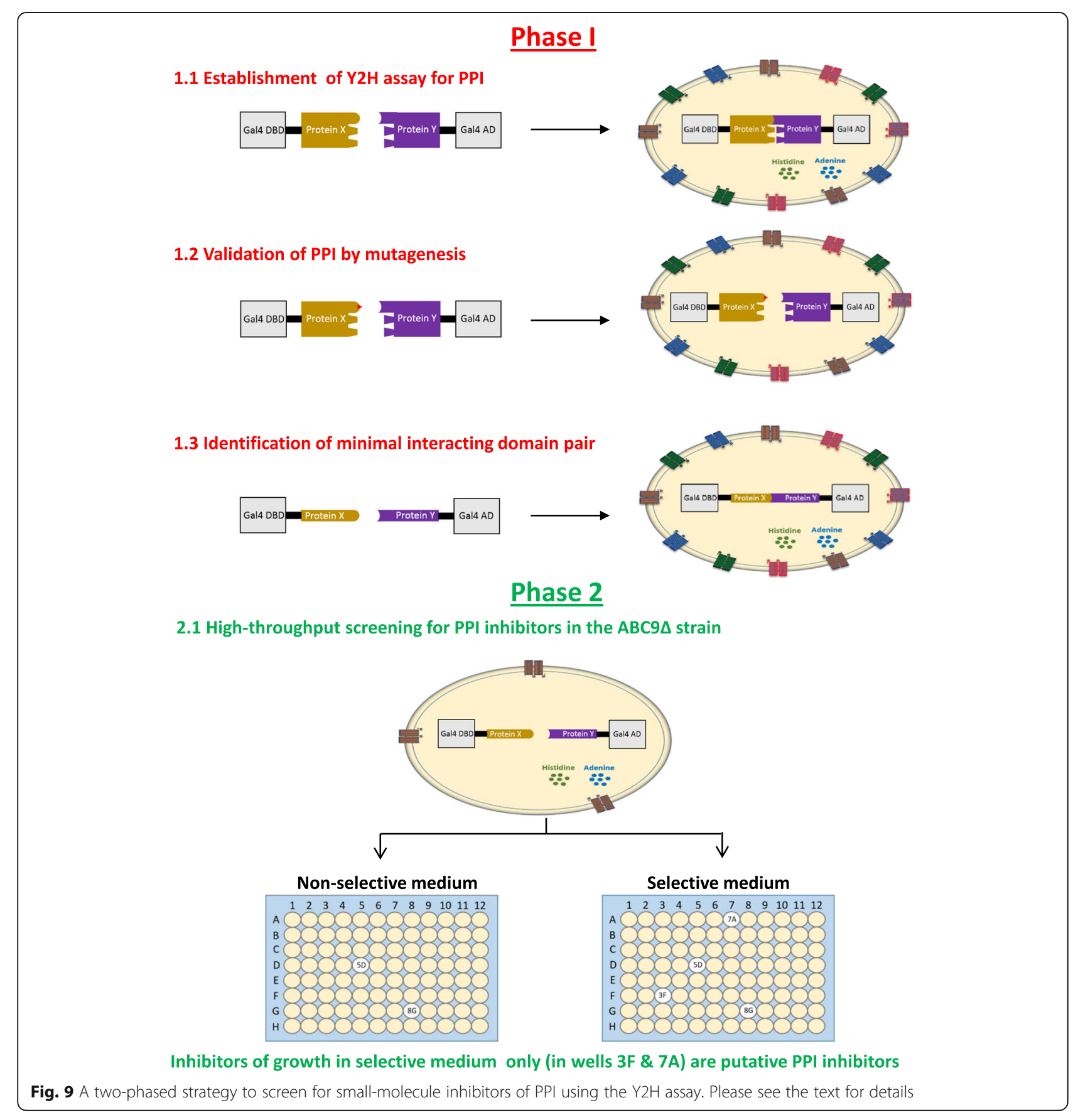


inhibit the growth of yeast cells only in selective media are putative PPI inhibitors and can be taken up for further investigation.

\section{Discussion}

By using the p53-Mdm2 interaction, which is amenable to small-molecule inhibition, as a model, we have developed and validated an improved version of the $\mathrm{Y} 2 \mathrm{H}$ assay that can be used in a high-throughput manner. To accomplish this, we made two crucial changes. Firstly, we used the minimal Mdm2-interacting domain in p53 instead of full-length p53. Secondly, we increased the permeability of yeast cells to small molecules by disabling $\mathrm{ABC}$ transporters.

By analyzing the p53 domains required for conferring resistance to inhibition by small molecules, we identified a hitherto unreported Mdm2-interacting segment overlapping with the highly conserved Box II region in the core domain of p53. Mdm2 inhibits the DNA binding activity of $\mathrm{p} 53$, but precisely how this occurs remains unknown. Our observations offer an explanation for this. As the DNA domain of p53 is engaged in interactions with Mdm2, p53-Mdm2 complexes may have a restricted ability to bind DNA. It will be interesting to test the effect of mutating segment 1 in the core domain of p53 on the Mdm2 inhibition of the DNA-binding activity of p53 and on the function and stability of p53 in mammalian cells.

Why do small-molecule inhibitors inhibit the full-length p53-Mdm2 interaction in mammalian cells but not in the $\mathrm{Y} 2 \mathrm{H}$ system? Since a good correlation between the strength of the p53-Mdm2 interaction in vitro and an ease of inhibition by small-molecule inhibitors in the $\mathrm{Y} 2 \mathrm{H}$ assay was observed, we suggest that there are factors in mammalian cells that compete with $\mathrm{Mdm} 2$ in binding to p53. Displacement of the transcriptional activation domain of $\mathrm{p} 53$ from $\mathrm{Mdm} 2$ by small-molecule inhibitors might create a binding site for proteins like p300, which are not present in yeast cells. Alternatively, posttranslational modifications in either p53 or Mdm2 in mammalian cells may weaken the p53-Mdm2 interaction. Our observations might explain why the p53-Mdm2 interaction is only partially inhibited by nutlin in vitro [35].

Complete inhibition of the p53 (1-52) interaction with Mdm2 by AMG232 and MI-773 at nanomolar concentrations suggests that the assay is highly sensitive. AMG232 was by far the most potent of the three inhibitors tested. This is consistent with its dissociation constant $(0.045$ $\mathrm{nM})$ being lower than that of MI-773 (62 $\mathrm{nM})$ and nutlin (90 nM). In contrast to nutlin, AMG232 and MI773 were able to inhibit the p53 (1-52)/Mdm2 interaction in wildtype cells. Nutlin thus appears to be a better substrate for yeast ABC transporters than MI-773 and AMG232. As many naturally occurring compounds, such as alkaloids, have an imidazole ring like nutlin, it is tempting to speculate that yeast $\mathrm{ABC}$ transporters have evolved to recognize such compounds. As human cells contain several ABC transporters, it will be interesting to test whether cotreatment of cancer cells with $\mathrm{ABC}$ transporter inhibitors improves the anti-cancer activity of nutlin.

We observed an excellent correlation between resistance of the p53-Mdm2 interaction to small-molecule inhibitors and the strength of the PPI in the in vitro binding assay. Binding of p53 (1-52/1-116) to Mdm2 is sensitive to nutlin in the $\mathrm{Y} 2 \mathrm{H}$ assay and is too weak to be detected in the binding assay. In contrast, the binding of p53 (1-143/1-160) is resistant to nutlin in the $\mathrm{Y} 2 \mathrm{H}$ assay and can be robustly detected in the binding assay. Binding of full-length p53 to Mdm2 was the strongest in the in vitro binding assay and resistant to nutlin in the $\mathrm{Y} 2 \mathrm{H}$ assay. It is worth highlighting that the expression of p53 was approximately 10-fold lower compared to other p53 variants in yeast cells and its resistance to small-molecule inhibitors in the $\mathrm{Y} 2 \mathrm{H}$ assay could be underestimated. Mutations in the Box II region of p53 (1-143) that render sensitivity to nutlin in the $\mathrm{Y} 2 \mathrm{H}$ assay also abolish its association with $\mathrm{Mdm} 2$ in the in vitro binding assay. We therefore propose that the $\mathrm{Y} 2 \mathrm{H}$ assay is not only useful in screening for PPI inhibitors but could also help to delineate domains involved in stabilizing PPIs.

\section{Conclusion}

In summary, by disabling several $A B C$ transporters in yeast, we developed a significantly improved $\mathrm{Y} 2 \mathrm{H}$ assay to screen for small-molecule inhibitors of PPIs. Using inhibitors of the p53-Mdm2 interaction to validate the $\mathrm{Y} 2 \mathrm{H}$ assay and demonstrate its suitability for highthroughput screening, we have also identified a novel putative Mdm2-binding site in the highly conserved Box II region of p53. The ease and economic nature of the assay, coupled with advantages of an in vivo-based system, makes it a valuable addition to the existing stockpile of in vitro tools to screen for PPI inhibitors.

\section{Methods \\ Plasmid construction}

The human p53 and Mdm2 ORF's were PCR amplified using Phusion polymerase (NEB, Ipswich, MA, USA) from plasmids encoding p53 cDNA and Mdm2 cDNA and cloned into pGADT7-AD and pGBKT7$\mathrm{BD}$, respectively, by gap-repair [36]. Plasmids were isolated from yeast transformants, rescued in $E$. coli and their integrity was confirmed by sequencing. Truncated p53 (1-52, 1-100, 1-116, 1-143, 1-160, 1-306, 1-363, 43-393) and Mdm2 (25-109, 1-125), and mutant p53 (F19A, Seg mutants and Box-V deletion $(\Delta 267-281)$ and $M d m 2(M 62 A)$ were generated using a similar approach. Plasmids streptavidin- 
binding peptide (SBP)-p53-pET22b (+) and HAMdm2-pET22b (+) were gifts from Dr Farid Ghadessy (p53 laboratory, Singapore). A list of plasmids used in the study is available in Additional file 14: Table S1.

\section{Yeast strain construction}

Derivatives of the yeast strain AH109 in the MATCHMAKER GAL4 Two-Hybrid system (Clontech Laboratories, CA, USA) were used in this study. ABC transporter-related genes were deleted by standard methods using PCR product-mediated homologous recombination. The ABC9 $\triangle$ strain was generated by crossing single deletion strains with each other. A list of yeast strains used in the study is available in Additional file 15: Table S2.

\section{Drug sensitivity test using the $\mathrm{Y} 2 \mathrm{H}$ assay}

( \pm )-Nutlin-3 (Cayman Chemicals, Ann Arbor, MI, USA) was dissolved in DMSO to make $50 \mathrm{mM}$ stock solution. MI-773 (MedChem Express Co. Ltd., China) and AMG232 (MedChem Express) were dissolved in DMSO to make $10 \mathrm{mM}$ stock solutions. Overnight yeast cultures were washed and then diluted to $\mathrm{OD}_{600}=0.2$ in 1 $\mathrm{mL}$ SD-Leu-Trp-His-Ade medium containing the desired concentration of the drug. The cultures were then placed in a $30{ }^{\circ} \mathrm{C}$ incubator with shaking for 3 days. Growth of the cultures was monitored by measuring the absorbance at $600 \mathrm{~nm}$ using the Implen Nanophotometer. This assay was also performed in a 96-well plate format with $200 \mu \mathrm{L}$ SD-Leu-Trp-His-Ade medium containing the desired concentration of the drug in duplicate. $\mathrm{OD}_{600}$ at different time points was measured using a Gen $5^{\mathrm{TM}}$ (BIO-TEK Instrument, Vermont, USA) microplate reader. The average of the two $\mathrm{OD}_{600}$ readings was calculated and used in the analysis.

\section{In vitro transcription-translation}

The SBP-p53 and HA-Mdm2 ORF's were amplified using SBP-p53-pET22b (+) and HA-Mdm2-pET22b (+) vectors as templates. Truncated (1-52, 1-116, 1-143, and $1-160$ ) and mutated p53 were generated by overlap PCR. These PCR products were used as substrates for in vitro translation (IVT) using PURExpress in vitro protein synthesis kit according to the instruction manual (NEB, Ipswich, MA, USA).

\section{Immunoprecipitation}

IVT-expressed wild-type, truncated, or mutant p53 were pre-cleared with Protein G beads (Invitrogen, Carlsbad, CA, USA), followed by incubation with IVT-expressed Mdm2 at room temperature for $1 \mathrm{~h}$. Anti-HA 3 F10 antibody (Roche Life Science, USA) at 1:200 dilution was added into the p53/Mdm2 mixture and incubated at $4{ }^{\circ} \mathrm{C}$ for $1 \mathrm{~h}$. Protein $\mathrm{G}$ beads were pre-blocked with $3 \%$
BSA/PBS and then added into the p53-Mdm2-anti-HA 3 F10 mixture. The beads were then washed once in $1 \times$ lysis buffer (50 mM Tris- $\mathrm{HCl} \mathrm{pH}$ 8.0, $5 \mathrm{mM}$ EDTA pH 7.4, $150 \mathrm{mM} \mathrm{NaCl}, 0.5 \% \mathrm{NP}-40$, and $1 \mathrm{mM}$ DTT), followed by two washes with $1 \times$ SNNTE buffer $(50 \mathrm{mM}$ Tris- $\mathrm{HCl} \mathrm{pH} 7.4,5 \mathrm{mM}$ EDTA $\mathrm{pH}$ 7.4, $5 \%$ sucrose, $1 \%$ $\mathrm{NP}-40,500 \mathrm{mM} \mathrm{NaCl}$, and $1 \mathrm{mM} \mathrm{DTT}$ ) and finally washed with $1 \times$ lysis buffer. Bound proteins were eluted by adding $40 \mu \mathrm{L}$ SDS-PAGE loading buffer to the tubes followed by incubation at $95{ }^{\circ} \mathrm{C}$ for 5 minutes.

\section{Western blot analysis}

Protein samples were resolved by electrophoresis on $12 \%$ SDS-PAGE gels and transferred onto nitrocellulose membranes. Blots were blocked with $5 \%$ milk in PBS/ $0.1 \%$ Tween 20. For p53, blots were probed with antip53 DO-1 antibody conjugated with horseradish peroxidase (1:1000; Santa Cruz Biotechnology, Santa Cruz, CA, USA). For Mdm2, the blot was probed with antiHA 3F10 antibody (1:2000; Roche Life Science, USA) followed by goat anti-rat HRP-conjugated antibody (1:5000; Santa Cruz Biotechnology). Blots were developed with ECL prime western blotting detection reagent (Amersham Pharmacia Biotech, USA).

\section{Additional files}

Additional file 1: Figure S1. Crystal structures of Mdm2 bound to p53 transactivation domain (17-29), nutlin, MI-773, and AMG232. (A) Mdm2 in magenta surface bound to the TAD1 of p53 (residues 17-29) in yellow ribbon with its three critical hydrophobic residues F19, W23, and L26 shown in sticks (PDB id: 1YCR) [17]. (B, C, D) Mdm2 in magenta surface bound to nutlin-3A (PDB id: 1RV1) [19], MI-773 (PDB id: 5TRF) [20], and AMG232 (PDB id: 4WT2) [21], respectively, in sticks. Images were generated using PyMOL (DeLano, W. L. The PyMOL Molecular Graphics System, DeLano Scientific, 2002). (EPS 6007 kb)

Additional file 2: Figure S2. Nutlin inhibits the p53 (1-52)/Mdm2 interaction in $A B C 3 \Delta$ cells. (A) Overnight cultures of AH109 yeast cells containing plasmids encoding either Gal4 AD-p53/Gal4 BD-Mdm2 in non-selective medium were washed in water and inoculated into selective medium at $\mathrm{OD}_{600}=0.2$ with nutlin at the indicated concentrations. Growth of the cultures was monitored by recording the absorbance at $600 \mathrm{~nm}$ at 0 , 32 , and $49 \mathrm{~h}$ following inoculation. (B) Overnight cultures of ABC3 $\Delta$ cells containing plasmids encoding either Gal4 AD-Csm1/Gal4 BD-Dsn1 or Gal4 AD-p53/Gal4 BD-Mdm2 in non-selective medium were washed in water and inoculated at $\mathrm{OD}_{600}=0.2$ into selective and non-selective medium containing DMSO or nutlin at the indicated concentrations. Growth of the cultures was monitored by recording the absorbance at $600 \mathrm{~nm}$ at the indicated time points. (C) Overnight cultures of $A B C 3 \Delta$ cells containing plasmids encoding either Gal4 AD-Csm1/Gal4 BD-Dsn1 or Gal4 AD-p53 $(1-52) / G a 14$ BD-Mdm2 in non-selective medium were washed in water and inoculated at $\mathrm{OD}_{600}=0.2$ into non-selective and selective medium containing DMSO or nutlin at the indicated concentrations. Growth of the cultures was monitored by recording the absorbance at $600 \mathrm{~nm}$ at the indicated time points. (EPS $1597 \mathrm{~kb})$

Additional file 3: Figure S3. M62A mutation in Mdm2 abolishes the nutlin sensitivity of the p53 (1-52)/Mdm2 (1-125) interaction. Overnight cultures of ABC3 $\Delta$ cells containing plasmids encoding either Gal4 AD-Csm1/ Gal4 BD-Dsn1 or Gal4 AD-p53 (1-52)/Gal4 BD-Mdm2 or Gal4 AD-p53 (1-52)/ Gal4 BD-Mdm2 (1-125) or Gal4 AD-p53 (1-52)/Gal4 BD-Mdm2 (1-125-M62A) 
in non-selective medium were washed in water and inoculated at $\mathrm{OD}_{600}=0.2$ into selective medium containing DMSO or nutlin at the indicated concentrations. Growth of the cultures was monitored by recording the absorbance at $600 \mathrm{~nm}$ at the indicated time points. (EPS $1261 \mathrm{~kb}$ )

Additional file 4: Figure S4. Nutlin is a substrate of the yeast $A B C$ transporter Pdr5. Overnight cultures of either wild-type or $p d r 5 \Delta$ or $p d r 1 \Delta$ or $p d r 3 \Delta$ or $p d r 5 \Delta p d r 1 \Delta p d r 3 \Delta$ cells containing plasmids encoding Gal4 AD-p53 (1-52)/Gal4 BD-Mdm2 were washed in water and inoculated at $\mathrm{OD}_{600}=0.2$ into selective medium containing DMSO or nutlin at the indicated concentrations. Growth of the cultures was monitored by recording the absorbance at $600 \mathrm{~nm}$ after $0,23,46$, and $71 \mathrm{~h}$ following inoculation. (EPS $1323 \mathrm{~kb}$ )

Additional file 5: Figure S5. $A B C$ transporters have distinct substrate specificities. (A) Overnight cultures of either wild-type or the different deletion strains, containing plasmids encoding Gal4 AD-p53 (1-52)/Gal4 BD-Mdm2, were washed in water and inoculated at $\mathrm{OD}_{600}=0.2$ into selective medium containing DMSO or nutlin at the indicated concentrations. Growth of the cultures was monitored by recording the absorbance at $600 \mathrm{~nm}$ after 0 (1), 24 (2), 44 (3), and $68 \mathrm{~h}$ (4) following inoculation. (B) Similar to A, but with rapamycin at the indicated concentrations. Absorbance measurements were performed after 0 (1), 21 (2), and $29 \mathrm{~h}$ (3). (EPS $1629 \mathrm{~kb}$ )

Additional file 6: Figure S6. $A B C$ transporters have distinct substrate specificities. Repeat of the experiment described in Additional file 5: Figure S5A. (EPS $1316 \mathrm{~kb}$ )

Additional file 7: Figure S7. Nutlin sensitivity of p53 (1-52)/Mdm2 interaction is enhanced in the $A B C 9 \Delta$ strain in comparison to $A B C 3 \Delta$ and wild-type strains. Overnight cultures of $A B C 3 \triangle$ or $A B C 9 \triangle$ cells containing plasmids encoding either Gal4 AD-Csm1/Gal4 BD-Dsn1 or Gal4 AD-p53 $(1-52) / G a l 4$ BD-Mdm2 in non-selective medium were washed in water and inoculated into selective medium at $\mathrm{OD}_{600}=0.2$. Growth of the cultures was monitored by recording the absorbance at $600 \mathrm{~nm}$ after 0 , 30, 48, and $72 \mathrm{~h}$ following inoculation. (EPS $1329 \mathrm{~kb}$ )

Additional file 8: Figure S8. The transactivation domain is required for the interaction of p53 (1-160) with Mdm2. Overnight cultures of AH109 yeast cells containing plasmids encoding either Gal4 AD-p53 (1-160)/Gal4 BD-Mdm2 or Gal4 AD-p53 (1-160-F19A)/Gal4 BD-Mdm2 or Gal4 AD-p53 (43-160)/Gal4 BD-Mdm2 in non-selective medium were washed in water and inoculated into selective and non-selective medium at $\mathrm{OD}_{600}=0.2 \mathrm{in}$ triplicate. Growth of the cultures was monitored by recording the absorbance at $600 \mathrm{~nm}$ after 0 (1), 19 (2), 28 (3), 44 (4), 51 (5), and 65 h (6) following inoculation. Average absorbance of the three cultures at different time points is indicated along with the error bars. (EPS $1397 \mathrm{~kb}$ )

Additional file 9: Figure S9. The Gal4 AD-full-length p53 fusion is expressed poorly compared to the other p53 variants. Protein extracts from logarithmically growing $A B C 9 \triangle$ cells containing plasmids encoding the indicated Gal4 AD- and Gal4 BD-fusion proteins were electrophoresed by SDS-PAGE and the p53 and Mdm2 proteins were detected by western blotting using anti-HA and anti-myc antibodies. Cdc28 served as a loading control. (EPS $24410 \mathrm{~kb}$ )

Additional file 10: Figure S10. Location of residues in Segments 1, 2 and 3 in the structure of p53's DNA-binding domain (DBD) complexed to DNA. p53's DBD (residues 95-293) is shown in green in the cartoon with the zinc atom shown as a cyan sphere. DNA (GGACATGTCCG) is shown in orange in the cartoon with blue sticks representing the bases. Segment 1 (F113 + G117-V122), Segment 2 (T123-A129), and Segment 3 (F134-V143) are shown in yellow, violet, and magenta, respectively. Image was generated using PyMOL (DeLano, W. L. The PyMOL Molecular Graphics System, DeLano Scientific, 2002) with the crystal structure of p53-DNA complex (PDB id: 2AHI) [34]. (EPS $1572 \mathrm{~kb})$

Additional file 11: Figure S11. Mutations in Segment 1 in p53's DNA-binding domain confer nutlin sensitivity to the p53 (1-143)/ Mdm2 interaction. Overnight cultures of $A B C 3 \Delta$ cells containing plasmids encoding either Gal4 AD-Csm1/Gal4 BD-Dsn1 or Gal4 AD-p53 (1-52)/Gal4 BD-Mdm2 or Gal4 AD-p53 (1-143)/Gal4 BD-Mdm2 or Gal4 AD-p53 (1-143-Seg1AA)/Gal4 BD-Mdm2 or Gal4 AD-p53 (1-143-Seg1BA)/Gal4 BD-Mdm2 or Gal4 AD-p53 (1-143-Seg1CA)/Gal4 BD-Mdm2 or p53 (1-143-Seg2AA)/Gal4 BD-Mdm2 or Gal4 AD-p53 (1-143-Seg2BA)/Gal4 BD-Mdm2 or Gal4 AD-p53 (1-143-Seg2CA)/Gal4
BD-Mdm2 in non-selective medium were washed in water and inoculated at $\mathrm{OD}_{600}=0.2$ into selective medium containing DMSO or nutlin at the indicated concentrations. Growth of the cultures was monitored by recording the absorbance at $600 \mathrm{~nm}$ after 0 (1), 20 (2), 27 (3), 44 (4), and 51 h (5) following inoculation. Residues replaced with alanine in the various mutants are indicated in red. Ends of the vertical bar indicate the $\mathrm{OD}_{600}$ values of the duplicate cultures. (A) Protein extracts from logarithmically growing $\mathrm{ABC} \triangle \Delta$ cells containing plasmids encoding the indicated Gal4 AD- and Gal4 BD-fusion proteins were electrophoresed by SDS-PAGE and the p53 and Mdm2 proteins were detected by western blotting analysis using anti-HA and anti-myc antibodies, respectively. Beta-actin served as a loading control. (EPS $19340 \mathrm{~kb}$ )

Additional file 12: Figure S12. The conserved Segment 1 in p53's DNA-binding domain promotes interaction with Mdm2. Amounts of Mdm2-HA immunoprecipitated in the samples from the binding reactions described in Fig. $8 \mathrm{c}$ were analyzed by western blotting using anti-HA antibody. (EPS $2200 \mathrm{~kb}$ )

Additional file 13: Figure S13. Mutations in Segment 1 in p53's DNA-binding domain does not affect nutlin sensitivity of the p53-FL/Mdm2 interaction. Overnight cultures of $A B C 3 \triangle$ cells containing plasmids encoding either Gal4 AD-Csm1/Gal4 BD-Dsn1 or Gal4 AD-p53 (1-52)/Gal4 BD-Mdm2 or Gal4 AD-p53/Gal4 BD-Mdm2 or Gal4 AD-p53-Seg1A/Gal4 BD-Mdm2 in non-selective medium were washed in water and inoculated at $\mathrm{OD}_{600}=0.2$ into selective medium containing DMSO or nutlin at the indicated concentrations. Growth of the cultures was monitored by recording the absorbance at $600 \mathrm{~nm}$ after 0 (1), 23 (2), 30 (3), 47 (4), and $54 \mathrm{~h}$ (5) following inoculation. Ends of the vertical bar indicate the $\mathrm{OD}_{600}$ values of the duplicate cultures. (EPS $1438 \mathrm{~kb}$ )

Additional file 14: Table S1. List of plasmids used in the study. (PDF $66 \mathrm{~kb}$ )

Additional file 15: Table S2. List of yeast strains used in the study. (PDF $111 \mathrm{~kb}$ )

\section{Abbreviations}

PPI: protein-protein interaction; SBP: streptavidin-binding peptide;

SD: synthetic dropout; $\mathrm{Y} 2 \mathrm{H}$ : yeast two-hybrid assay

\section{Acknowledgements}

We would like to thank Ms Feiqi Cao and Ms Devika Warrier for assistance with the yeast two-hybrid assays and Dr Farid Ghadessy and Ms Jia Wei Siau for advice regarding in vitro binding assay and for p53/Mdm2 plasmids.

\section{Funding}

This work was supported by core budget from the Bioinformatics Institute (BII) and Industrial Alignment Fund (IAF311013) from the Agency for Science, Technology and Research (A-STAR), Singapore.

\section{Availability of data and materials}

All reasonable requests for strains and plasmids used in the research will be fulfilled, provided that a written agreement is executed in advance between the Bioinformatics Institute and the requester (and their affiliated institution). Such requests should be directed to the corresponding author. The data supporting the conclusions of this article are included within the article and its additional files.

\section{Authors' contributions}

JHW designed and performed experiments, analyzed the data, and helped in writing of the manuscript. AM performed experiments and helped in artwork. MFS performed experiments. HMS helped in experimental design. CV and DPL helped with experimental design and writing of the manuscript. PA led the whole project, performed experiments, analyzed the data, and wrote the manuscript. All authors have read and approved the final manuscript.

Ethics approval and consent to participate Not applicable 


\section{Consent for publication}

Not applicable

\section{Competing interests}

The authors declare that they have no competing interests.

\section{Publisher's Note}

Springer Nature remains neutral with regard to jurisdictional claims in published maps and institutional affiliations.

\begin{abstract}
Author details
'Bioinformatics Institute, 30 Biopolis Street, \#07-01, Matrix, Singapore 138671, Singapore. ${ }^{2}$ Department of Pharmacy, National University of Singapore, National University of Singapore 18 Science Drive 4, Singapore 117543, Singapore. ${ }^{3}$ Department of Biological Sciences, National University of Singapore, 14 Science Drive, Singapore 117543, Singapore. ${ }^{4}$ Nanyang Technological University, School of Biological Sciences, 50 Nanyang Drive, Singapore 637551, Singapore. ${ }^{5}$ The p53 Laboratory, 8A Biomedical Grove, Singapore 138648, Singapore.
\end{abstract}

Received: 5 June 2017 Accepted: 19 October 2017

Published online: 09 November 2017

\section{References}

1. Stumpf MP, Thorne T, de Silva E, Stewart R, An HJ, Lappe M, Wiuf C. Estimating the size of the human interactome. Proc Natl Acad Sci U S A. 2008;105:6959-64

2. Whitty A, Kumaravel G. Between a rock and a hard place? Nat Chem Biol. 2006:2:112-8.

3. Arkin MR, Tang Y, Wells JA. Small-molecule inhibitors of protein-protein interactions: progressing toward the reality. Chem Biol. 2014;21:1102-14.

4. Clackson T, Wells JA. A hot spot of binding energy in a hormone-receptor interface. Science. 1995;267:383-6.

5. Sheng C, Dong G, Miao Z, Zhang W, Wang W. State-of-the-art strategies for targeting protein-protein interactions by small-molecule inhibitors. Chem Soc Rev. 2015;44:8238-59.

6. Fields S, Song O. A novel genetic system to detect protein-protein interactions. Nature. 1989;340:245-6.

7. Schwikowski B, Uetz P, Fields S. A network of protein-protein interactions in yeast. Nat Biotechnol. 2000;18:1257-61.

8. Rolland T, Tasan M, Charloteaux B, Pevzner SJ, Zhong Q, Sahni N, Yi S, Lemmens I, Fontanillo C, Mosca R, et al. A proteome-scale map of the human interactome network. Cell. 2014;159:1212-26.

9. Leanna CA, Hannink M. The reverse two-hybrid system: a genetic scheme for selection against specific protein/protein interactions. Nucleic Acids Res. 1996:24:3341-7.

10. Bennett MA, Shern JF, Kahn RA. Reverse two-hybrid techniques in the yeast Saccharomyces cerevisiae. Methods Mol Biol. 2004;261:313-26.

11. Huang J, Schreiber SL. A yeast genetic system for selecting small molecule inhibitors of protein-protein interactions in nanodroplets. Proc Natl Acad Sci U S A. 1997;94:13396-401.

12. Kato-Stankiewicz J, Hakimi I, Zhi G, Zhang J, Serebriiskii I, Guo L, Edamatsu $\mathrm{H}$, Koide H, Menon S, Eckl R, et al. Inhibitors of Ras/Raf-1 interaction identified by two-hybrid screening revert Ras-dependent transformation phenotypes in human cancer cells. Proc Natl Acad Sci U S A. 2002;99: 14398-403.

13. Rezwan M, Auerbach D. Yeast "N"-hybrid systems for protein-protein and drug-protein interaction discovery. Methods. 2012;57:423-9.

14. Vousden KH, Lane DP. p53 in health and disease. Nat Rev Mol Cell Biol. 2007:8:275-83

15. Kan Z, Jaiswal BS, Stinson J, Janakiraman V, Bhatt D, Stern HM, Yue $P$, Haverty PM, Bourgon R, Zheng J, et al. Diverse somatic mutation patterns and pathway alterations in human cancers. Nature. 2010;466:869-73.

16. Momand J, Zambetti GP, Olson DC, George D, Levine AJ. The mdm-2 oncogene product forms a complex with the p53 protein and inhibits p53mediated transactivation. Cell. 1992;69:1237-45.

17. Kussie PH, Gorina S, Marechal V, Elenbaas B, Moreau J, Levine AJ, Pavletich NP. Structure of the MDM2 oncoprotein bound to the p53 tumor suppressor transactivation domain. Science. 1996;274:948-53.
18. Shangary S, Wang S. Small-molecule inhibitors of the MDM2-p53 proteinprotein interaction to reactivate p53 function: a novel approach for cancer therapy. Annu Rev Pharmacol Toxicol. 2009;49:223-41.

19. Vassilev LT, Vu BT, Graves B, Carvajal D, Podlaski F, Filipovic Z, Kong N, Kammlott U, Lukacs C, Klein C, et al. In vivo activation of the p53 pathway by small-molecule antagonists of MDM2. Science. 2004;303:844-8.

20. Wang S, Sun W, Zhao Y, McEachern D, Meaux I, Barriere C, Stuckey JA, Meagher JL, Bai L, Liu L, et al. SAR405838: an optimized inhibitor of MDM2p53 interaction that induces complete and durable tumor regression. Cancer Res. 2014;74:5855-65.

21. Sun D, Li Z, Rew Y, Gribble M, Bartberger MD, Beck HP, Canon J, Chen A, Chen X, Chow D, et al. Discovery of AMG 232, a potent, selective, and orally bioavailable MDM2-p53 inhibitor in clinical development. J Med Chem. 2014;57:1454-72.

22. Dawson R, Muller L, Dehner A, Klein C, Kessler H, Buchner J. The N-terminal domain of p53 is natively unfolded. J Mol Biol. 2003;332:1131-41.

23. Wallace M, Worrall E, Pettersson S, Hupp TR, Ball KL. Dual-site regulation of MDM2 E3-ubiquitin ligase activity. Mol Cell. 2006;23:251-63.

24. Poyurovsky MV, Katz C, Laptenko O, Beckerman R, Lokshin M, Ahn J, Byeon IJ, Gabizon R, Mattia M, Zupnick A, et al. The C terminus of p53 binds the Nterminal domain of MDM2. Nat Struct Mol Biol. 2010;17:982-9.

25. Stepanov A, Nitiss KC, Neale G, Nitiss JL. Enhancing drug accumulation in Saccharomyces cerevisiae by repression of pleiotropic drug resistance genes with chimeric transcription repressors. Mol Pharmacol. 2008;74:423-31.

26. Sarkar S, Shenoy RT, Dalgaard JZ, Newnham L, Hoffmann E, Millar JB, Arumugam P. Monopolin subunit Csm1 associates with MIND complex to establish monopolar attachment of sister kinetochores at meiosis I. PLoS Genet. 2013;9:e1003610.

27. Wei SJ, Joseph T, Chee S, Li L, Yurlova L, Zolghadr K, Brown C, Lane D, Verma C, Ghadessy F. Inhibition of nutlin-resistant HDM2 mutants by stapled peptides. PLoS One. 2013;8:e81068.

28. Paumi CM, Chuk M, Snider J, Stagljar I, Michaelis S. ABC transporters in Saccharomyces cerevisiae and their interactors: new technology advances the biology of the ABCC (MRP) subfamily. Microbiol Mol Biol Rev. 2009;73:577-93.

29. Decottignies A, Grant AM, Nichols JW, de Wet H, Mclntosh DB, Goffeau A. ATPase and multidrug transport activities of the overexpressed yeast $A B C$ protein Yor1p. J Biol Chem. 1998;273:12612-22.

30. Rogers B, Decottignies A, Kolaczkowski M, Carvajal E, Balzi E, Goffeau A. The pleitropic drug ABC transporters from Saccharomyces cerevisiae. J Mol Microbiol Biotechnol. 2001;3:207-14.

31. Khakhina S, Johnson SS, Manoharlal R, Russo SB, Blugeon C, Lemoine S, Sunshine AB, Dunham MJ, Cowart LA, Devaux F, Moye-Rowley WS. Control of plasma membrane permeability by ABC transporters. Eukaryot Cell. 2015; 14:442-53.

32. Korotchkina LG, Leontieva OV, Bukreeva El, Demidenko ZN, Gudkov AV, Blagosklonny MV. The choice between p53-induced senescence and quiescence is determined in part by the mTOR pathway. Aging (Albany NY) 2010;2:344-52.

33. Wei SJ, Joseph T, Sim AY, Yurlova L, Zolghadr K, Lane D, Verma C, Ghadessy F. In vitro selection of mutant HDM2 resistant to Nutlin inhibition. PLoS One. 2013;8:e62564

34. Kitayner M, Rozenberg H, Kessler N, Rabinovich D, Shaulov L, Haran TE, Shakked Z. Structural basis of DNA recognition by p53 tetramers. Mol Cell. 2006:22:741-53.

35. Funston G, Goh W, Wei SJ, Tng QS, Brown C, Jiah Tong L, Verma C, Lane D, Ghadessy F. Binding of translationally controlled tumour protein to the $\mathrm{N}$ terminal domain of HDM2 is inhibited by nutlin-3. PLoS One. 2012;7:e42642.

36. Oldenburg KR, Vo KT, Michaelis S, Paddon C. Recombination-mediated PCRdirected plasmid construction in vivo in yeast. Nucleic Acids Res. 1997;25: $451-2$. 